\title{
Comparative Review on Thin Film Growth of Iron-Based Superconductors
}

\author{
Yoshinori Imai ${ }^{1, *,+}$, Fuyuki Nabeshima ${ }^{2, *,+}$ and Atsutaka Maeda ${ }^{2}$ \\ 1 Department of Physics, Tohoku University, Sendai 980-8578, Japan \\ 2 Department of Basic Science, University of Tokyo, Tokyo 153-8902, Japan; cmaeda@mail.ecc.u-tokyo.ac.jp \\ * Correspondence: imai@tohoku.ac.jp (Y.I.); nabeshima@maeda1.c.u-tokyo.ac.jp (F.N.); \\ Tel.: +81-22-795-5570 (Y.I.); +81-3-5454-6763 (F.N.) \\ + These authors contributed equally to this work.
}

Academic Editor: Yoshikazu Mizuguchi

Received: 3 April 2017; Accepted: 3 July 2017; Published: 7 July 2017

\begin{abstract}
Since the discovery of the novel iron-based superconductors, both theoretical and experimental studies have been performed intensively. Because iron-based superconductors have a smaller anisotropy than high- $T_{\mathrm{c}}$ cuprates and a high superconducting transition temperature, there have been a lot of researchers working on the film fabrication of iron-based superconductors and their application. Accordingly, many novel features have been reported in the films of iron-based superconductors, for example, the fabrication of the epitaxial film with a higher $T_{\mathrm{c}}$ than bulk samples, the extraction of the metastable phase which cannot be obtained by the conventional solid state reaction, and so on. In this paper, we review the progress of research on thin film fabrications of iron-based superconductors, especially the four categories: $\operatorname{LnFeAs}(\mathrm{O}, \mathrm{F})(L n=$ Lanthanide), $A E \mathrm{Fe}_{2} \mathrm{As}_{2}(A E=$ Alkaline-earth metal), $\mathrm{FeCh}(\mathrm{Ch}=$ Chalcogen $)$, and FeSe monolayer. Furthermore, we focus on two important topics in thin films of iron-based superconductors; one is the substrate material for thin film growth on the iron-based superconductors, and the other is the whole phase diagram in $\mathrm{FeSe}_{1-x} \mathrm{Te}_{x}$ which can be obtained only by using film-fabrication technique.
\end{abstract}

Keywords: iron-based superconductors; film fabrication; phase diagram

\section{Introduction}

Research on iron-based superconductors is one of the most exciting topics in recent condensed matter physics. Triggered by the discovery of a superconductivity in $\mathrm{LaFeP}(\mathrm{O}, \mathrm{F})$ [1] and $\mathrm{LaFeAs}(\mathrm{O}, \mathrm{F})[2]$, both theoretical and experimental studies on iron-based superconductors have been performed intensively. For many researchers, it is very surprising that the material including iron, which is a typical magnetic element that is generally considered not to be a "good friend" of a superconductivity, at the key position in the crystal shows a superconductivity with a superconducting transition temperature, $T_{\mathrm{C}}$, above $20 \mathrm{~K}$. Within only a few months since the report on $\mathrm{LaFeAs}(\mathrm{O}, \mathrm{F})[2]$, the substitution of other Lanthanide atoms is found to raise $T_{\mathrm{c}}$ rapidly, and the value of $T_{\mathrm{c}}$ for iron-based superconductors increased up to $\sim 56 \mathrm{~K}$ in $\operatorname{SmFeAs}(\mathrm{O}, \mathrm{F})$ [3] with a ZrCuSiAs-type structure which was the same crystal structure as $\operatorname{LaFeAs}(\mathrm{O}, \mathrm{F})$ [2]. Now, many iron-based superconductors have been discovered [4], and the iron-based superconductors attain the positions of a new class of high- $T_{\mathrm{c}}$ superconductor next to cuprates. The superconductivity of iron-based superconductors cannot be explained theoretically by a conventional electron-phonon interaction, similar to a case of cuprates, and thus there is an almost complete consensus that the superconductivity in both these two systems is due to an unconventional origin. The band calculation at early stages [5-9] revealed that the Fermi surface of iron-based superconductors was constructed from five orbitals of iron. One of the most important features of iron-based superconductors is that these are multi-band 
compounds, where the Fermi surface consists of hole pockets around $\Gamma$-point and electron- pockets around M-points in most of iron-based superconductors. Thus, the origin of the unconventional superconductivity has been considered to be a pairing mediated by a spin [8,9] and/or orbital [10-12] fluctuations related to the nesting between the hole and electron Fermi surface. However, in the FeSe monolayer films [13] and $A_{x} \mathrm{Fe}_{y} \mathrm{Se}_{2}(A=$ Alkaline metal) [14,15], the only electron pockets are observed in their Fermi surface. These cannot be explained by the simple $s_{ \pm}$superconductivity which was proposed as a superconductivity mediated by a spin fluctuation in iron-based superconductors at early stages. At this moment, there are no theories by which all the novel features of various iron-based superconductors can be explained, and the iron-based superconductors which exhibit an intimate interplay of orbital, spin, and lattice degrees of freedom provide an interesting platform for researches in the fundamental physics.

The iron-based superconductors are attractive materials also in terms of their application, because they have a smaller anisotropy than cuprate superconductors and large values of $T_{\mathrm{C}}$ and the upper critical field, $B_{\mathrm{c} 2}$. Thus, the extensive works on the film growth of iron-based superconductors have been performed. Here, we will briefly describe the importance and the merit for the film fabrication. The film fabrication technique is very important for not only application but also fundamental study. The tensile/compressive strain can be induced into a film by using a lattice mismatch between a film and a substrate. This is an anisotropic pressure effect in contrast to the hydrostatic pressure. Thus, the strain effect in films and the hydrostatic pressure provide a complementary information. The film fabrication is also a powerful tool for searching new materials. The process of the film fabrication is a thermodynamically non-equilibrium one, which is effective for obtaining a meta-stable phase. In addition, by utilizing the superlattice technique, a material with a new stacking structure can be designed. As can be expected easily, the film technique is necessary for an application like the Josephson junctions and the superconducting quantum interference devices (SQUID). The fabrication of the Josephson junction is very important also for a fundamental study. The phase-sensitive experimental techniques were developed to determine the symmetry of the pair state in cuprate superconductors. Indeed, the observation of the half-integer quantum flux in the $\mathrm{YBa}_{2} \mathrm{Cu}_{3} \mathrm{O}_{7}$ films on the tri-crystal (100) $\mathrm{SrTiO}_{3}$ substrates provides the direct evidence for the $d$-wave superconductivity [16,17]. As described above, the film technique is very important for both the application and the fundamental study. Thus, the researches related on the film synthesis of the iron-based superconductors have also been performed from the early period by many groups. There were some difficulties in the film fabrication of these materials at the early period and these techniques for the film fabrication have much advanced in these 9 years. Today, in some materials of iron-based superconductors, the epitaxial thin films with higher $T_{\mathrm{c}}$ 's than bulk samples can be obtained. Especially, in monolayer film of FeSe, which is one of the iron-based superconductors with a $T_{\mathrm{C}}$ of $8 \mathrm{~K}$ [18] in bulk samples, the superconducting-like gap structure was observed at higher temperatures than boiling point of liquid nitrogen in some in situ measurements. In addition, the fabrication of the device, Josephson junctions and SQUID, by using the films of iron-based superconductors has also been reported. As the remarkable development has been made in the film growth of iron-based superconductors in this manner, in this paper, we overview the progress of the studies on the film fabrication of iron-based superconductors from our viewpoint at first, while there have already been some nice review articles [4,19-26]. Subsequently, we focus on the two topics; one is the substrate material in the thin film growth of iron-based superconductors. The substrate material is one of the key parameters for the thin film fabrication. Interestingly, in thin films of 1111, 122, and 11, the highest $T_{\mathrm{c}}$ for each systems is commonly obtained in the case of the same substrate material, $\mathrm{CaF}_{2}$, although the in-plane lattice constants largely differ from each other. We would like to compare the superconducting and structural properties in 1111, 122, and 11 films on $\mathrm{CaF}_{2}$ and to discuss the reason why the films on $\mathrm{CaF}_{2}$ show superconductivity with high $T_{\mathrm{c}}$. The other is the whole phase diagram for iron chalcogenide superconductor, $\mathrm{FeSe}_{1-x} \mathrm{Te}_{x}$, which can be obtained only by utilizing the film-fabrication technique. Recently, extensive studies on iron selenide, FeSe, have been 
performed, because FeSe exhibits an orbital and/or electronic nematic order without accompanying antiferromagnetic order at low temperatures, unlike other iron-based superconductors. However, in a Te-substituted iron selenide, $\mathrm{FeSe}_{1-x} \mathrm{Te}_{x}$, bulk sample, it is well-known that a phase separation occurs at $0.1 \leq x \leq 0.4$ [27], which prevents systematic studies on this material. We compare the phase diagrams of $\mathrm{FeSe}_{1-x} \mathrm{Te}_{x}$ bulk samples and films, and review the reported results on $\mathrm{FeSe}_{1-x} \mathrm{Te}_{x}$ films with a whole range of $x$.

\section{Historical Overview of Film Fabrication of Iron-Based Superconductors}

In iron-based superconductors, the studies on the film fabrication of iron-based superconductors are mainly performed in the four systems; (1) $\mathrm{LnFeAsO}$ (1111-system); (2) $A E \mathrm{Fe}_{2} \mathrm{As}_{2}$ (122-system); (3) FeCh (11-system); and (4) FeSe mono-layer film (11-system ML).We overview the progress of the researches on the film fabrication of these four kinds of materials.

\subsection{1-System}

Hiramatsu et al. reported the successful fabrication of an epitaxial thin film of LaFeAsO by a pulsed laser deposition (PLD) method, which is the first report on the film fabrication of the iron-based superconductors [28].They attempted some methods (simple PLD, post-thermal annealing, and reactive solid-phase epitaxy, which were used for the with the same crystal structure) for obtaining $\mathrm{LaFeAsO}_{1-x} \mathrm{~F}_{x}$ film, and successfully fabricated LaFeAsO epitaxial thin films on $\mathrm{MgO}, \mathrm{MgAl}_{2} \mathrm{O}_{4}$, and $(\mathrm{La}, \mathrm{Sr})(\mathrm{Al}, \mathrm{Ta}) \mathrm{O}_{3}$ (LSAT) substrates only by a simple PLD method. The key growth condition was the excitation source of the laser ablation, which the second harmonics of a neodymium-doped yttrium aluminum garnet (Nd-YAG) laser with $\lambda=532 \mathrm{~nm}$ was suitable for the film growth of this system. The obtained LaFeAsO epitaxial thin film has slightly larger $a$-axis and $c$-axis lengths than those of $\mathrm{LaFeAsO}$ bulk samples [2]. In the electrical resistivity measurement of this film, the kink anomaly is observed around $150 \mathrm{~K}$ as is polycrystalline samples, which is due to the structural and spin density wave transitions [2,29]. Unfortunately, however, the superconducting $\mathrm{LaFeAsO}_{1-x} \mathrm{~F}_{x}$ film cannot be obtained even by using the $\mathrm{LaFeAsO}_{0.9} \mathrm{~F}_{0.1}$ target. In their latter work [30], the authors recognized that the difficulty of thin film growth of $L n \mathrm{Fe} P n \mathrm{O}$ ( $P n$ : Pnictogen) may come from the crystal structure containing two different anions, because it is difficult to control their stoichiometry, especially for thin film growth. Indeed, it was only recently that the superconducting 1111 film fabrication via in situ PLD method was reported by using the diffusion of fluorine into the film from the $\mathrm{CaF}_{2}$ substrate [31].

Almost simultaneously, Backen et al. also reported the fabrication of the superconducting $\mathrm{LaFeAsO}_{1-x} \mathrm{~F}_{x}$ polycrystalline film by the ex-situ process [32]. The amorphous-like films were deposited on $\mathrm{MgO}$ and $\mathrm{LaAlO}_{3}$ (LAO) substrates at room temperature by PLD method, and then the superconducting films were obtained by the post-annealing in an evacuated silica-glass tube at high temperatures. The obtained film was a polycrystalline one, and showed only the onset of superconducting transition at $\sim 11 \mathrm{~K}$ in the temperature dependence of resistivity. Then, the superconducting films which showed a zero resistivity around $T_{\mathrm{c}}$ of bulk samples were successfully synthesized by revising the annealing condition $[33,34]$. The film synthesis by using the post-annealing out of the deposition chamber has already been summarized by Haindl et al. [26], and we reviewed the epitaxial thin film fabrication by the in situ process in the followings.

The technical breakthrough is the successful fabrication of $\mathrm{NdFeAsO}$ (Nd1111) epitaxial thin film by molecular beam epitaxy (MBE) [35]. Although the growth window is very narrow, the single crystalline film of $\mathrm{NdFeAsO}$ can be reproducibly fabricated on GaAs substrate. One year later, Kawaguchi et al. reported the successful fabrication of superconducting single crystalline films of F-doped Nd1111 by MBE process and found that the growth time, $t_{g}$, has a crucial importance for the superconducting properties of resultant films [36]. When $t_{g} \leq 3 \mathrm{~h}$, the single crystalline films with almost no impurity phases were obtained and did not show a superconductivity, which were similar to their previous report [35]. However, when $t_{g} \geq 4 \mathrm{~h}$, the $\mathrm{Nd} 1111$ phase was still major one, and some impurity phases ( $\mathrm{NdOF}, \mathrm{Fe}_{2} \mathrm{O}_{3}$, and $\mathrm{FeAs}$ ) appeared. The formation of NdOF phase became 
apparent especially in films grown for $t_{g}=5-6 \mathrm{~h}$, which showed a clear superconducting transition ( $T_{\mathrm{c}}^{\text {onset }} \approx 48 \mathrm{~K}, T_{\mathrm{c}}^{\text {zero }} \approx 42 \mathrm{~K}$ for $t_{g}=6 \mathrm{~h}$ ). Superconductivity emerged with a formation of an impurity phase of $\mathrm{NdOF}$, which suggested that this impurity phase played a role in a dopant of fluorine into a film. However, the understanding for the deposition process was not so complete at this moment. In this process, both $\mathrm{Nd}$ and $\mathrm{F}$ were provided from the raw material of $\mathrm{NdF}_{3}$ and it was impossible to control these contents independently [36]. This difficulty was overcome by the co-evaporation of Ga. Kawaguchi et al. found that the use of Ga as a getter were effective for controlling a amount of fluorine [37]. Even when $t_{g} \leq 1 \mathrm{~h}$, a superconductivity is observed in the NdFeAsO film on which a NdOF layer was intentionally grown. On the other hand, Naito et al. reported that the SmFeAsO (Sm1111) film on $\mathrm{CaF}_{2}$ substrate with a cap layer of $\mathrm{SmF}_{3}$ fabricated by $\mathrm{MBE}$ showed a superconducting transition at $\sim 56 \mathrm{~K}$ which was slightly higher than $T_{\mathrm{c}}$ of $\operatorname{SmFeAs}(\mathrm{O}, \mathrm{F})$ bulk samples [38,39]. Although $\mathrm{CaF}_{2}$ was firstly used by Tsukada et al. as a substrate material in the film growth of $\mathrm{FeSe}_{1-x} \mathrm{Te}_{x}$ [40], which will be discussed later, the enhancement of $T_{\mathrm{c}}$ also in $\mathrm{Sm} 1111$ film on $\mathrm{CaF}_{2}$ [38] shows that $\mathrm{CaF}_{2}$ is efficient for the thin film growth of other iron-based superconductors. In these reports, the extra cap layer is necessary to obtain a superconducting 1111 film, which prevents an application of 1111 film for a sandwich-type junction [36,38]. The one-step growth method which does not need the extra cap layer for obtaining a superconductivity was established by using $L n \mathrm{~F}_{3}(\mathrm{Ln}=\mathrm{Sm}, \mathrm{Nd})$ or $\mathrm{FeF}_{2}$ as a fluorine source [41-43].Naito et al. pointed out the importance of the fluorine source in the film growth of 1111 and proposed a four kinds of fluorine sources [41]; (1) F diffused from the fluoride substrate $\left(\mathrm{CaF}_{2}, \mathrm{SrF}_{2}, \mathrm{BaF}_{2}\right)$; (2) $\mathrm{F}$ from $L n \mathrm{~F}_{3}$ codeposited; (3) $\mathrm{F}$ from $\mathrm{FeF}_{n}(n=2,3)$ codeposited; and (4) molecular $F_{2}$ gas. Although method (4) is not realistic, the other three methods are effective for supplying F to the films in the MBE process. While methods (2) and (3) are shown above [42,43], a superconductivity appeared in the $\mathrm{SmFeAsO}$ film on the $\mathrm{BaF}_{2}$ substrate, in which the fluorine was provided by the diffusion from the substrates [41]. On the other hand, highly textured $\mathrm{NdFeAs}(\mathrm{O}, \mathrm{F})$ thin films have been grown on ion beam assisted deposition(IBAD)- $\mathrm{MgO} / \mathrm{Y}_{2} \mathrm{O}_{3} / \mathrm{Hastelloy}$ substrates by $\mathrm{MBE}$, which is well known as the one of the most practical methods to obtain the textured film on the metallic substrate [44].

While some kinds of the fluorine sources are proposed in the MBE process, it has been considered for a long time that in the PLD process the fluorine can be doped into a film only from a target, which prevents an in situ growth of a superconducting 1111 film via PLD. Recently, Haindl et al. reported the in situ growth of superconducting Sm1111 films on $\mathrm{CaF}_{2}$ substrates via PLD by using the F diffusion from the substrate [31]. The $\operatorname{SmFeAs}(\mathrm{O}, \mathrm{F})$ films fabricated on $\mathrm{BaFe}_{2} \mathrm{As}_{2}-\mathrm{buffered} \mathrm{MgO}$ substrates by using the $\mathrm{SmFeAsO} \mathrm{O}_{0.9} \mathrm{~F}_{0.1}$ polycrystalline target had almost the same lattice parameters as those of SmFeAsO bulk samples and showed an anomaly, which is due to a structural transition [39], around $150 \mathrm{~K}$ and no superconductivity in the temperature dependence of resistivity. These results indicate that the F content in this film is insufficient. On the other hand, the Sm1111 film on CaF substrate deposited from the same target shows a superconductivity at $\sim 40 \mathrm{~K}$. The $c$-axis length is comparable to that of Sm1111 film on $\mathrm{CaF}_{2}$ fabricated by MBE [38,41-43] and is slightly larger than that of Sm1111 bulk samples [39]. This indicates that the fluorine diffused from the substrate is doped into a film, which induces a superconductivity as is the case of MBE [41]. Although the fluoride $\mathrm{CaF}_{2}$ is generally thought to be stable, the fluorine in the substrate can diffuse into a film in the film fabrication process. The similar fluorine diffusion was proposed also in the film growth of $\mathrm{FeSe}_{0.5} \mathrm{Te}_{0.5}[45,46]$, which was confirmed from these above superconductivity in 1111 films [31,41].

In summary, the superconducting single crystalline thin films can be fabricated by MBE and PLD methods. A fluorine source is a key issue in the film growth of this material. At this moment, however, the precise control of the amount of fluorine is difficult in the 1111 film fabrication, and the doping dependence of the structural and superconducting properties has not been clarified in the 1111 film yet. 


\subsection{2-System}

Soon after the first report on the La1111 film fabrication [28], Hiramatsu et al. reported the successful fabrication of an epitaxial superconducting thin film of $\mathrm{Sr}(\mathrm{Fe}, \mathrm{Co})_{2} \mathrm{As}_{2}$ (Co-doped $\left.\mathrm{Sr} 122\right)$ on LSAT substrate via PLD using Nd:YAG laser [30]. In this report, they pointed out that the Co doping into Fe site was easier than $\mathrm{K}$ doping into Sr site in 122 or $\mathrm{F}$ doping into $\mathrm{O}$ site in 1111 because of low vapor pressure of $\mathrm{Co}$ and that the film growth of Co-doped Sr122 which contains only one anion (As) would be easier than a mixed-anion compound 1111 containing two or three kinds of anions (As, O, and F). Later, Choi et al. reported that the Co-doped Sr122 superconducting film could be prepared even by using not Nd:YAG laser but $\mathrm{KrF}$ laser [47]. In the thin film growth of 122 and 11, the Nd:YAG laser is not indispensable unlike the 1111 system, and there are many reports on the film fabrication via PLD using KrF or ArF lasers. In addition, Hiramatsu et al. showed that a superconductivity could be induced in the parent Sr122 film by exposing to the air [48]. When the parent Sr122 films were exposed to the dry oxygen, dry nitrogen, and dry carbon dioxide gas, these treated films did not show a superconductivity. On the other hand, the Sr122 film after the exposure to the water vapor showed a superconductivity around $25 \mathrm{~K}$. Therefore, this novel superconductivity was considered to be induced by the water vapor and the detailed mechanism of this phenomena is not unclear at this moment. Anyway, this means that Sr122 films are very sensitive to the air and the moisture and that they are unstable in the atmosphere. These features of Sr122 films are not favorable for its application.

Katase et al. reported the first successful fabrication of $\mathrm{Ba}(\mathrm{Fe}, \mathrm{Co})_{2} \mathrm{As}_{2}$ (Co-doped $\mathrm{Ba} 122$ ) superconducting epitaxial film by PLD and showed that Co-doped Ba122 film was much more stable in the air than Co-doped Sr122 film [49]. So, hereafter, we focus on the Ba122 system in this review. In addition, Lee et al. fabricated Co-doped Ba122 films on four kinds of [001]-tilt-(100)-SrTiO 3 bicrystal substrates whose misorientation angles are $3^{\circ}, 6^{\circ}, 9^{\circ}$, and $24^{\circ}$ [50]. These films showed a superconductivity above $20 \mathrm{~K}$, and the critical current density across [001] tilt grain boundaries, $J_{\mathrm{gb}}$, of $\mathrm{Ba}(\mathrm{Fe}, \mathrm{Co})_{2} \mathrm{As}_{2}$ is strongly depressed at $\theta=9^{\circ}, 24^{\circ}$, which is similar to high- $T_{\mathrm{c}}$ cuprates. At the similar period, lida et al. [51] showed the comparative study on the Co-doped Ba122 epitaxial thin films fabricated on four kinds of oxide substrates $\left(\mathrm{SrTiO}_{3}(\mathrm{STO}), \mathrm{LAO}, \mathrm{LSAT}\right.$, and $\mathrm{YAlO}_{3}(\mathrm{YAO})$ ) by the same condition. The highest $T_{\mathrm{c}}$ of $24.5 \mathrm{~K}$ was obtained in the film on STO which has the smallest $a$-axis length among four films. In addition, they insisted that the values of $T_{\mathrm{c}}$ can be scaled by the ratio of $c / a$. The similar results were reported in 11 films [52]. For improving the superconducting properties of the resultant films, many groups tried a variety of approaches. Lee et al. proposed that the introduction of STO or $\mathrm{BaTiO}_{3}$ (BTO) thin buffer layers on LAO and LSAT substrates was effective for improving the film quality [53]. The Co-doped Ba122 films on these buffer layers showed a sharp superconducting transition ( $T_{\mathrm{c}}^{\text {onset }} \approx 22.8 \mathrm{~K}, T_{\mathrm{c}}^{\text {zero }} \approx 21.5 \mathrm{~K}, \Delta T_{\mathrm{c}} \approx 1.3 \mathrm{~K}$ ), and the self-field critical current density, $J_{\mathrm{c}}$, was as high as $4.5 \mathrm{MA} / \mathrm{cm}^{2}$ at $4.2 \mathrm{~K}$. lida et al. found another useful buffer layer, that is, metal iron with a body-centered-cubic (bcc) structure whose bond length along [110] direction was close to that along [100] direction of Ba122 [54-56]. Indeed, by introducing the bcc-Fe buffer layer with thickness of $\sim 15 \mathrm{~nm}$, the obtained values of $T_{\mathrm{c}}$ for Co-doped Ba122 films on LSAT and MgO substrates increased by $\sim 2-4 \mathrm{~K}$ compared to those of films on bare these substrates. Additionally, the observed self-field $J_{\mathrm{C}}$ in the Co-doped Ba122 films on the Fe buffer layer was $0.45 \mathrm{MA} / \mathrm{cm}^{2}$ at $12 \mathrm{~K}$, which was several times higher than that of the film on the bare LSAT substrate [57]. On the other hand, Katase et al. demonstrated that the Co-doped Ba122 films with high $T_{\mathrm{c}}$ and $J_{\mathrm{c}}$ could be grown without any buffer layers only by the optimization of the PLD deposition condition [58]. They improved the phase purity of the PLD target and the homogeneity of the substrate temperature, and fabricated the Co-doped Ba122 film on LSAT substrate with $T_{\mathrm{c}}^{\text {onset }}$ of $22.6 \mathrm{~K}, \Delta T_{\mathrm{c}}$ of $1.1 \mathrm{~K}$, and $J_{\mathrm{c}}$ of $2-10 \mathrm{MA} / \mathrm{cm}^{2}$ at $3 \mathrm{~K}$. These values of $T_{\mathrm{C}}$ and $J_{\mathrm{C}}$ are comparable to those of films on STO or iron buffer layers. In addition, by applying the superior film fabrication technique, Katase et al. successfully fabricated the Josephson junction of the Co-doped Ba122 film on the bicrystal LSAT substrate [58] and SQUID [59], which is the first demonstration of these devices in the films of the iron-based superconductors. Around 
the same time, Schmidt et al. reported the successful fabrication of a multi layer Josephson junction (superconductor-normal metal-superconductor, SNS) consisting of the Co-doped Ba122, PbIn and $\mathrm{Au}$ [60]. The Co-doped Ba122 film can be grown more easily than 1111-system and many groups reported the film growths of this material. These studies were performed for obtaining the high $J_{\mathrm{c}}$ or fabricating junctions and devices, and there were no reports on the growth of Co-doped Ba122 films on oxide substrates with $T_{\mathrm{c}}$ higher than $\sim 26 \mathrm{~K}$ which was the highest $T_{\mathrm{c}}$ of Co-doped Ba122 bulk samples [61,62]. Iida et al. fabricated the Co-doped Ba122 films on fluoride $A E F_{2}(A E=\mathrm{Ca}$, $\mathrm{Sr}, \mathrm{Ba}$ ) substrates [63], which had been used in the film growth of 11- [40] and 1111-systems [38]. The in-plane lattice parameter of Co-doped Ba122 film on $\mathrm{CaF}_{2}$ is smaller than those of Co-doped Ba122 bulk samples [61,62], which indicates that the film received the in-plane compressive strain. The obtained superconducting transition temperature is $T_{\mathrm{c}}^{\text {onset }} \approx 28 \mathrm{~K}$ [63], which is higher than the highest $T_{\mathrm{C}}$ in Co-doped Ba122 bulk samples [61,62].

A superconductivity can be induced in $\mathrm{BaFe}_{2} \mathrm{As}_{2}$ by the doping at the sites other than the iron site. The bulk samples of $\mathrm{Ba}_{1-x} \mathrm{~K}_{x} \mathrm{Fe}_{2} \mathrm{As}_{2}$ (K-doped Ba122) show a superconductivity at $x=0.16-1$, which the highest $T_{\mathrm{c}}$ is $\sim 40 \mathrm{~K}$ around $x=0.4[62,64]$. The $\mathrm{K}$-doped Ba122 epitaxial single crystalline films with whole range of $x$ were successfully fabricated on LAO, $\mathrm{MgO}, \mathrm{LAO}, \mathrm{STO}$, and r-cut $\mathrm{Al}_{2} \mathrm{O}_{3}$ substrates by MBE [65-67] and by PLD [68]. The highest $T_{\mathrm{c}}$ of K-doped Ba122 films was obtained around $x=0.4$ and its value is as high as $\sim 38 \mathrm{~K}$ [65-67], which are comparable with the highest $T_{\mathrm{C}}$ of K-doped Ba122 bulk samples [61,62]. Because K-doped Ba122 has high $T_{\mathrm{C}}$ [62] and low anisotropy [69-71] compared with Co-doped Ba122, K-doped Ba122 is considered to be a prospective material for an application. However, the film deposition of K-doped Ba122 is very difficult because of the high vapor pressure of potassium and there are fewer papers on the K-doped Ba122 films than Co-doped Ba122.

In $\mathrm{BaFe}_{2} \mathrm{As}_{2}$, the chemical pressure is introduced by a partial substitution of $\mathrm{P}$ for As, which also induces a superconductivity [72]. In $\mathrm{BaFe}_{2}\left(\mathrm{As}_{1-x} \mathrm{P}_{x}\right)_{2}$ (P-doped Ba122) bulk samples, a superconductivity appeared at $x=0.2-0.7$ and the highest $T_{\mathrm{C}}$ is as high as $\sim 31 \mathrm{~K}$ around $x=0.33$ [62]. The P-doped Ba122 epitaxial thin films were successfully fabricated by PLD [73,74] and MBE [75]. While $T_{\mathrm{c}}$ of these films [73-75] is comparable to that of bulk sample [62], it is noteworthy that the obtained $J_{\mathrm{c}}$ is extremely high. Sakagami et al. showed that $J_{\mathrm{C}}$ of P-doped Ba122 films strongly depended on the $\mathrm{Ba} / \mathrm{Fe}$ ratio, and that the highest self-field $J_{\mathrm{c}}$ was as high as $10 \mathrm{MA} / \mathrm{cm}^{2}$ at $4.2 \mathrm{~K}$ [75]. They speculated that the nano-particles of Fe or Fe-based compounds which could not be detected by XRD were acted as pinning centers since the highest $J_{c}$ was obtained in the iron-rich film [75]. Later, from scanning transmission electron microscopy (STEM) measurement, Sato et al. reported that the vertical dislocations along the $c$-axis existed in the P-doped Ba122 film fabricated by PLD and that these served as strong vortex-pinning centers [76]. The existence of these vertical dislocations is probably the origin of the isotropic and large $J_{\mathrm{c}}$ in the P-doped Ba122 film. The other striking feature is the dependence of $J_{\mathrm{c}}$ on the misorientation angle, $\theta_{\mathrm{GB}}$, in P-doped Ba122 films fabricated on bicrystal substrates. Sakagami et al. [75] demonstrated that $J_{\mathrm{c}}$ across a grain boundary in the P-doped Ba122 film on the $\mathrm{MgO}$ bicrystal substrate with $\theta_{\mathrm{GB}}=24^{\circ}$ exceeded $1 \mathrm{MA} / \mathrm{cm}^{2}$ at $4.2 \mathrm{~K}$. This value is much larger than those of $\mathrm{YBa}_{2} \mathrm{Cu}_{3} \mathrm{O}_{7-\delta}$ [77] and Co-dope Ba122 [78], which strongly indicates that the P-dope Ba122 has a prospective material for an application of superconducting tapes or wires.

In Ba122, there are a few reports on the superconductivity induced by using distinctive features of the film technique. One is the electron doping by a partial substitution of $L n$ for Ba [79]. One of the characteristics of Ba122 is that a superconductivity can be induced by the substitution at any of three sites [62]. Superconductivity appears by the substitution of $\mathrm{K}$ at $\mathrm{Ba}$ site, which introduces hole carriers in Ba122 [80]. However, the electron doping by substituting the Ba site cannot be realized by a conventional solid state reaction. Katase et al. were succeeded in the electron doping by the atomic substitution at Ba site via a non-equilibrium film growth process. They successfully fabricated $\mathrm{Ba}_{1-x} \mathrm{La}_{x} \mathrm{Fe}_{2} \mathrm{As}_{2}(x=0-0.44)$ epitaxial thin films on $\mathrm{MgO}$ substrates by PLD method. In the temperature dependence of resistivity, an anomaly was observed around $135 \mathrm{~K}$ in $\mathrm{BaFe}_{2} \mathrm{As}_{2}$ film, which 
was well known behavior related to a structural/magnetic transition in $\mathrm{BaFe}_{2} \mathrm{As}_{2}$ bulk sample [61,62]. The characteristic temperature, $T_{\text {anom }}$, rapidly decreases with increasing $x$. The zero resistivity was observed at $\sim 5 \mathrm{~K}$ for the film with $x=0.08$, where $T_{\text {anom }}$ still remained at $\sim 72 \mathrm{~K}$. $T_{\mathrm{c}}^{\text {onset }}$ reached a maximum value of $22.4 \mathrm{~K}$ at $x=0.13$, where $T_{\text {anom }}$ just disappeared. $T_{\mathrm{C}}$ monotonically decreased with increasing $x$. At $x=0.44$, there was no superconductivity. Both the La substitution for Ba and the Co substitution of Fe is expected to introduce the electron carriers into Ba122. Indeed, in Co-doped Ba122 bulk single crystals, the evolution of the Fermi surface due to the electron doping was observed in the angle-resolved photoemission spectroscopy [81]. Since electronic states at the Fermi level are occupied predominantly by five $3 \mathrm{~d}$ orbitals of $\mathrm{Fe}$ in the iron-based superconductors, $T_{\mathrm{C}}$ of $\mathrm{Ba}_{1-x} \mathrm{La}_{x} \mathrm{Fe}_{2} \mathrm{As}_{2}$ is expected to be rather higher than that of Co-doped Ba122. Surprisingly, $T_{\mathrm{c}}$ of $\mathrm{Ba}_{1-x} \mathrm{La}_{x} \mathrm{Fe}_{2} \mathrm{As}_{2}$ is much lower than that of K-doped Ba122 and is comparable to that of Co-doped Ba122. This indicated that $T_{\mathrm{C}}$ was sensitive to the kinds of dominant carriers rather than the doping site. The other is the tensile-strain-induced superconductivity in the $\mathrm{BaFe}_{2} \mathrm{As}_{2}$ film [82]. Engelmann et al. fabricated the $\mathrm{BaFe}_{2} \mathrm{As}_{2}$ epitaxial films with different film thicknesses on Fe-buffered $\mathrm{MgAl}_{2} \mathrm{O}_{4}$ substrates with a spinel structure. It is no doubt that the parent $\mathrm{BaFe}_{2} \mathrm{As}_{2}$ bulk material is semi-metal and does not show a superconductivity [61,62]. In $\mathrm{BaFe}_{2} \mathrm{As}_{2}$ films with thickness less than $30 \mathrm{~nm}$, the in-plane lattice parameters are slightly larger than that of bulk sample, which shows that the tensile strain was introduced into the $\mathrm{BaFe}_{2} \mathrm{As}_{2}$ film. The tensile-strained $\mathrm{BaFe}_{2} \mathrm{As}_{2}$ films showed a superconductivity with $T_{\mathrm{C}}$ of 7-10 K. With increasing thickness, the in-plane lattice parameter got close to that of $\mathrm{BaFe}_{2} \mathrm{As}_{2}$ bulk sample and a superconductivity disappeared. Here, the important thing is that they used the same target of $\mathrm{BaFe}_{2} \mathrm{As}_{2}$ in the film fabrication and that the carrier concentration and the substrate material were not changed. These results suggest that a fine structure around the iron, which is sensitive to the changes of lattice parameters, has a crucial importance in the iron-based superconductors.

\subsection{1-System}

The first report of thin film growth of FeSe was made by Han et al. [83], which firstly appeared in the arXiv. They used off-stoichiometric $\mathrm{FeSe}_{1-x}$ polycrystals as the targets and STO, LSAT and LAO as substrates, and grew films via a PLD method. The highest $T_{\mathrm{C}}$ value of $T_{\mathrm{C}}^{\text {onset }} \sim 12 \mathrm{~K}$ was obtained for films on LAO substrates with $x=0.88$, while $T_{\mathrm{c}}^{\text {zero }}$ values were below $4 \mathrm{~K}$. They also pointed out difficulties of growing superconducting FeSe films because of its narrow growth window. Soon after Han et al., Wu et al. reported fabrication of FeSe and $\mathrm{FeSe}_{1-x} \mathrm{Te}_{x}$ films [84] (The publication was earlier than Han's paper). Their films show strong thickness dependence of $T_{\mathrm{C}}$ values. Although $T_{\mathrm{C}}$ increases with increasing film thickness, even films with thickness of $1 \mu \mathrm{m}$ did not have good superconducting properties compared with bulk samples.

After these papers, many research groups reported the thin film growth of FeSe and related compounds one after another [85-91]. Among them, some reported the enhancement of $T_{\mathrm{C}}$ in $\mathrm{FeSe}_{1-x} \mathrm{Te}_{x}[87,88]$. In particular, Bellingeri et al., who had already reported the enhanced $T_{\mathrm{c}}$ in $\mathrm{FeSe}_{1-x} \mathrm{Te}_{x}$ on STO, investigated thickness dependence in detail, and found that $T_{\mathrm{C}}$ strongly depends on thickness and a film on LAO with thickness of $200 \mathrm{~nm}$ shows $T_{\mathrm{C}}^{\text {onset }}$ of $21 \mathrm{~K}\left(T_{\mathrm{C}}^{\text {zero }}=19 \mathrm{~K}\right)$, which is 1.5 times higher than that of bulk $\mathrm{FeSe}_{1-x} \mathrm{Te}_{x}$ [92]. They demonstrated that $T_{\mathrm{c}}$ of $\mathrm{FeSe}_{0.5} \mathrm{Te}_{0.5}$ are correlated with $a$-axis length of the films and contraction of the $a$-axis length results in the enhancement of $T_{C}$, which is also the case with FeSe $[85,93,94]$. They estimated local structural parameters of the films from relative intensity of $\mathrm{XRD}$ and found that the $T_{\mathrm{C}}$ values increase as the $\mathrm{Ch}$-Fe-Ch bond angle reaches to that of the regular tetrahedron as expected by the empirical law concerning relations between structural parameters and $T_{\mathrm{C}}$ values [95,96]. Interestingly, thickness dependence of the $a$-axis length shows non-monotonic behavior; as the films become thick, the $a$-axis length of the films firstly becomes shorter and reaches minimum at $200 \mathrm{~nm}$ and then slowly becomes long. This non-monotonic dependence of lattice constants on thickness is also observed for FeSe on $\mathrm{CaF}_{2}[93,94]$. Bellingeri et al. inferred that the Volmer-Weber growth mode should be related to this non-monotonic thickness dependence and the contraction of $a$-axis length [92]. 
Control of lattice strain is particularly important for the growth of $\mathrm{FeSe}_{1-x} \mathrm{Te}_{x}$ films because $T_{\mathrm{c}}$ can be enhanced due to compression of $a$-axis length as Bellingeri et al. demonstrated [92]. The simplest way to achieve this is to utilizing epitaxial strain due to lattice mismatch between the film and the substrate. However, these attempts were not successful except for a few cases such as FeSe on LAO [97] and FeSe monolayer films on STO [98]. Imai et al. reported the growth of $\mathrm{FeSe}_{0.5} \mathrm{Te}_{0.5}$ films on eight different oxide substrates, and they found that $a$-axis length and the superconducting properties of the grown films are nothing to do with in-plane lattice parameters of the substrates [52]. Indeed, the films with best superconducting properties, are on $\mathrm{LAO}$ and $\mathrm{MgO}$, whose the lattice misfit parameter $M \equiv\left(a_{\text {sub }}-3.798\right) / a_{\text {sub }}=-0.21 \%$ and $9.82 \%$, respectively. It should be noted that Bellingeri et al. reported positive correlation between the in-plane lattice parameters of the films and the substrates [99], while their films were not coherently strained to the underlying substrates. The discrepancy between the observed results may result from the difference of the growth temperatures; Bellingeri et al. grew the films at much higher temperature of $550^{\circ} \mathrm{C}$ than Imai et al. $\left(300^{\circ} \mathrm{C}\right)$ [52].

One of the causes for which there is no correlation between in-plane lattice parameters of films and substrates may be chemical reaction at the interface between the film and the substrate $[52,100]$ (other possible causes are discussed in Section 3). Imai et al. also performed TEM observations of the cross-sections of the films [52]. Films on $\mathrm{LAO}$ and $\mathrm{MgO}$, which show the best superconducting properties, have clear interface between substrate and film. On the other hand, films on YSZ, which had worst superconducting properties, have amorphous-like layer at the interface. In addition, oxygen penetrates into films from substrates. These results clearly demonstrated that chemical reaction at the interface between the substrate and the film and oxygen penetration into the films have a bad influence on the superconducting properties of $\mathrm{FeSe}_{1-x} \mathrm{Te}_{x}$ films.

Consequently, various materials are tried as substrates and buffer layers in order to prevent oxygen penetration. Tsukada et al. focused on non-oxide substrates, and grew $\mathrm{FeSe}_{0.5} \mathrm{Te}_{0.5}$ films on $\mathrm{CaF}_{2}$ [40]. The grown films have shorter $a$-axis length compared with oxide substrates, and $T_{\mathrm{c}}$ increases to above $15 \mathrm{~K}$, higher than bulk value. Such a short $a$-axis length is not due to a simple coincidence to the lattice parameters of $\mathrm{CaF}_{2}$ because $a_{\text {sub }} / \sqrt{2}=3.863 \AA>a_{\text {film }}$. Detailed cross-sectional TEM observations suggests that chemical reaction at the interface between substrate and film will be related to the strong compression of $a$-axis length $[45,46]$. A belt-shaped pattern is observed near the interface between the substrate and the film in $\mathrm{CaF}_{2}$ in a TEM image, where Se penetrates from the films. Studies of composition dependence revealed that the compression of $a$-axis length is prominent for FeSe, while FeTe on $\mathrm{CaF}_{2}$ have almost the same $a$-axis length as bulk FeTe [101]. These results indicate the relation between compression of $a$ in $\mathrm{FeSe}_{1-x} \mathrm{Te}_{x}$ on $\mathrm{CaF}_{2}$ and chemical reaction at the interface between substrate and film. A possible explanation for the shortened $a$-axis length of the films on $\mathrm{CaF}_{2}$ is that at the beginning of the growth the initial several layers of $\mathrm{FeSe}_{1-x} \mathrm{Te}_{x}$ have shorter $a$-axis length due to the Se deficiency and then, the subsequent layers of $\mathrm{FeSe}_{1-x} \mathrm{Te}_{x}$ grow epitaxially on the initial layers with keeping the shorter $a$-axis length.

Having demonstrated the effectiveness of the use of the Fe-buffer layer in the growth of Co-doped $\mathrm{BaFe}_{2} \mathrm{As}_{2}$ thin films [54,55], Iida et al. also used Fe-buffered $\mathrm{MgO}$ substrates for the growth of $\mathrm{FeSe}_{0.5} \mathrm{Te}_{0.5}$ [102]. Then, they observed enhanced superconducting transition temperature of $T_{\mathrm{c}}^{\text {onset }}=$ $17.7 \mathrm{~K}$, indicating that the use of Fe-buffer is also effective to obtain high $T_{\mathrm{c}} \mathrm{FeSe}_{1-x} \mathrm{Te}_{x}$.

$\mathrm{Si}$ et al. found that $\mathrm{CeO}_{2}$ buffer is also effective for the growth of $\mathrm{FeSe}_{1-x} \mathrm{Te}_{x}$ [103]. $\mathrm{CeO}_{2}$ is a commonly used buffer layer for high $T_{\mathrm{c}}$ cuprates, and it has the in-plane lattice parameter of $a / \sqrt{2} \sim 3.83 \AA$, similar to that of $\mathrm{FeSe}_{1-x} \mathrm{Te}_{x}$. They observed $T_{\mathrm{c}}^{\text {onset }} \sim 20 \mathrm{~K}$ and $T_{\mathrm{c}}^{\text {zero }} \sim 18 \mathrm{~K}$ in $\mathrm{FeSe}_{1-x} \mathrm{Te}_{x}$ films not only on $\mathrm{CeO}_{2}$-buffered YSZ but also on Rolling Assisted Biaxially Textured Substrate (RABiTS) with $\mathrm{CeO}_{2}$ on top. Realization of enhanced superconductivity in $\mathrm{FeSe}_{1-x} \mathrm{Te}_{x}$ on coated conductors would be a great step forward the superconducting tape application of these materials.

Recently, the use of $\mathrm{FeSe}_{1-x} \mathrm{Te}_{x}$ itself as a buffer layer was reported by Molatta et al. [104] The growth window of superconducting $\mathrm{FeSe}_{1-x} \mathrm{Te}_{x}$ films is narrow, and although films grown at 
much higher temperatures does not show superconductivity, they have better texture with high reproducibility, to which Molatta et al. paid attention. They deposited thin $\mathrm{FeSe}_{1-x} \mathrm{Te}_{x}$ seed layer at $400{ }^{\circ} \mathrm{C}$ on $\mathrm{MgO}$, and subsequently lowered the temperature of the substrates and deposited another $\mathrm{FeSe}_{1-x} \mathrm{Te}_{x}$ layer. In this way, they succeeded in obtaining wider growth window $\left(240-320^{\circ} \mathrm{C}\right)$ compared with films without seed layer $\left(300-320^{\circ} \mathrm{C}\right)$ and superconducting thin films with reproducible high $T_{\mathrm{C}}$ over $17 \mathrm{~K}$.

The growth of $\mathrm{FeSe}_{1-x} \mathrm{Te}_{x}$ films by other techniques than PLD or MBE has also been reported. Tkachenko et al. reported the sputtering growth of FeSe thin films on $\mathrm{SrTiO}_{3}$ and $\mathrm{LaAlO}_{3}$ with $T_{\mathrm{c}}^{\text {onset }} \sim 10 \mathrm{~K}, T_{\mathrm{c}}^{\text {zero }} \sim 8 \mathrm{~K}[105]$. In contrast to FeSe [105,106], FeSe ${ }_{1-x} \mathrm{Te}_{x}$ films with $T_{\mathrm{c}}^{\text {zero }}$ comparable to that of bulk samples seem to be difficult to be obtained by sputtering techniques $[107,108]$. There are also attempts to grow FeSe films by electrochemical techniques [109-114] and chemical vapor deposition techniques $[115,116]$. However, $T_{\mathrm{c}}^{\text {zero }}$ comparable to that of bulk samples has not been obtained or the resistivity was not measured in these films.

The critical current properties of $\mathrm{FeSe}_{1-x} \mathrm{Te}_{x}$ thin films was first reported by Eiseterer et al. [117]. They investigated $J_{\mathrm{c}}$ properties of $\mathrm{FeSe}_{1-x} \mathrm{Te}_{x}$ thin films on LAO with $T_{\mathrm{c}}$ above $19 \mathrm{~K}$. Then they obtained $J_{\mathrm{c}}>0.8 \mathrm{MA} / \mathrm{cm}^{2}\left(\right.$ at $4.5 \mathrm{~K}$ and $\mu_{0} H=0 \mathrm{~T}$ ) and $J_{\mathrm{c}}>0.1 \mathrm{MA} / \mathrm{cm}^{2}$ (at $4.5 \mathrm{~K}$ and $\mu_{0} H=5 \mathrm{~T}$ ), which are much higher than those of sulfur-doped FeTe thin films that had been reported before [118]. They also found correlated pinning parallel to the film plane (parallel to the $a b$ plane) in all the measured samples, and they speculated that this could be intrinsic in nature. Iida et al. also observed the similar angular dependence of $J_{\mathrm{c}}$ in $\mathrm{FeSe}_{1-x} \mathrm{Te}_{x}$ films on Fe-buffered $\mathrm{MgO}$ [102]. They investigated electric field-current density curves of the films in detail, and concluded that these behavior are due to the intrinsic pinning in $\mathrm{FeSe}_{1-x} \mathrm{Te}_{x}$ [119]. On the other hand, Braccini et al. reported nearly isotropic $J_{\mathrm{c}}$ in $\mathrm{FeSe}_{1-x} \mathrm{Te}_{x}$ thin films on $\mathrm{CaF}_{2}$, which showed $J_{\mathrm{c}}>1 \mathrm{MA} / \mathrm{cm}^{2}$ in self field at $4 \mathrm{~K}$ [120]. Therefore $J_{\mathrm{c}}$ properties of $\mathrm{FeSe}_{1-x} \mathrm{Te}_{x}$ films are considered to depend strongly on the substrates. $\mathrm{FeSe}_{1-x} \mathrm{Te}_{x}$ has remarkable $J_{c}$ properties under magnetic fields and has potential for high-field magnet applications. Si et al. reported that $\mathrm{FeSe}_{1-x} \mathrm{Te}_{x}$ films both on $\mathrm{CeO}_{2}$-buffered $\mathrm{YSZ}$ and on RABiTS show $J_{\mathrm{c}}>0.1 \mathrm{MA} / \mathrm{cm}^{2}$ at $4.2 \mathrm{~K}$ and $\mu_{0} H=30 \mathrm{~T}$ [103]. In order to further improve $J_{c}$ properties, introduction of artificial pinning center should be needed. Indeed, Ozaki et al. reported enhancement in $T_{\mathrm{c}}$ and $J_{\mathrm{c}}$ by proton irradiation, which is considered to be due to local strain around the defects and pinning by the defects, respectively [121].

There are some reports on thin film growth of iron chalcogenides which are not available in bulk crystals. Below we describe three topics: (i) superconducting FeTe thin films; (ii) $\mathrm{FeSe}_{1-x} \mathrm{Te}_{x}$ films with compositions where bulk crystals are not available due to phase separation; and (iii) fabrication of superlattice films based on Fe chalcogenides.

FeTe shows an antiferromagnetic phase transition accompanied by structural transition at approximately $70 \mathrm{~K}$, and does not exhibit superconductivity. However, there are some reports on the growth of superconducting FeTe thin films [122-125]. Han et al. reported superconductivity in FeTe thin films with $T_{\mathrm{c}}^{\text {onset }}$ of $13 \mathrm{~K}$ for the first time [122]. They speculated that the superconductivity is due to tensile strain of the films. On the other hand, Si et al. argued that oxygen incorporation is crucial for the superconductivity in FeTe films [123]. They compared FeTe films grown in vacuum $\left(<2 \times 10^{-6}\right.$ Torr $)$ and oxygen $\left(\sim 1 \times 10^{-4}\right.$ Torr $)$, and demonstrated that films grown in oxygen shows better superconducting properties. Nie et al. investigated X-ray absorption spectra and showed that the superconducting samples exhibit Fe with a nominal 3+ valence [124]. Han et al. performed the composition analysis by the scanning electron microscopy and energy dispersive analysis of X-ray. However the oxygen composition in the film was not evaluated because a large amount of oxygen from the oxide substrate was detected. Therefore it is possible that the superconducting FeTe films of Han et al. also contained some amount of oxygen.

These results suggests oxygen incorporation is the key for superconducting FeTe. Interestingly, bulk FeTe samples do not show superconductivity even if they are annealed in oxygen. This suggests that thin-film growth may be another key for superconducting FeTe. However, because 
superconducting FeTe films can have both longer and shorter $a$-axis length [124], the mechanism seems not simple like the strain scenario by Han et al. [122], whereas the superconducting properties of FeTe may be largely affected by the lattice strain, suggested by thermal expansion measurements [126]. Physical properties of the superconducting FeTe are similar to those of $\mathrm{FeSe}_{1-x} \mathrm{Te}_{x}$ : (1) strain largely affects the $T_{\mathrm{C}}$ values [126]; (2) importance of both $n$ - and $p$-type carriers for the superconductivity, suggested by the Hall effect $[40,127,128]$; (3) huge $B_{c 2}(>30 \mathrm{~T})[122,123,129]$. These may suggest that the mechanism of the superconductivity is the same for both FeTe and $\mathrm{FeSe}_{1-x} \mathrm{Te}_{x}$.

It is well-known that $\mathrm{FeSe}_{1-x} \mathrm{Te}_{x}$ bulk samples with $0.1<x<0.4$ are not available due to phase separation [27]. Thin film deposition will enable the synthesis of a materials with metastable phase because it involves crystal growth in a thermodynamically non-equilibrium state. The first attempt to obtain single phase $\mathrm{FeSe}_{1-x} \mathrm{Te}_{x}$ bulk samples with $0.1<x<0.4$ has already been reported in 2009 by Wu et al. using a PLD method [84]. However their films with $0.1<x<0.4$ showed much broader $00 l$ reflection peaks in the X-ray diffraction than the films with other composition, which suggests non-uniform substitution of Te to Se sites. Successful suppression of phase separation in $\mathrm{FeSe}_{1-x} \mathrm{Te}_{x}$ was reported independently by Zhuang et al. and Imai et al. around the same time [130,131]. They both reported the thin film growth of $\mathrm{FeSe}_{1-x} \mathrm{Te}_{x}$ on $\mathrm{CaF}_{2}$ with $0.1<x<0.4$ via PLD. Importantly, the highest $T_{\mathrm{C}}$ was obtained at $x=0.2-0.4$, compositions where bulk samples cannot be obtained. Imai et al. reported that $T_{\mathrm{c}}$ reaches $22.3 \mathrm{~K}$ for $T_{\mathrm{C}}^{\text {onset }}$ and $21.7 \mathrm{~K}$ for $T_{\mathrm{c}}^{\text {zero }}$ at $x=0.3$, highest value among $\mathrm{FeSe}_{1-x} \mathrm{Te}_{x}$ samples at ambient pressure except for monolayer FeSe films on STO [132]. Suppression of phase separation is also possible with LAO substrates; in this case the optimal composition is $x=0.4$ [133]. These results demonstrate that the optimal composition, $x_{\mathrm{opt}}$, of $\mathrm{FeSe}_{1-x} \mathrm{Te}_{x}$ is in the phase separation region. Imai et al. also found that $T_{\mathrm{C}}$ rapidly changes at $x$ around $x_{\mathrm{opt}}$, and the composition dependence of $T_{\mathrm{C}}$ is different from a simple dome-shaped one unlike other iron-based superconductors [131], which will be a hint for understandings of superconductivity in these materials. Details on this topic are described in Section 4.

Since FeSe consists of conducting planes alone, novel materials can be artificially grown by depositing FeSe and another non-superconducting material alternately. Research on intercalated FeSe revealed that $T_{\mathrm{C}}$ may depend on the interlayer distance between each FeSe layer and $T_{\mathrm{C}}$ increases over $40 \mathrm{~K}$ with samples with large interlayer distance $[134,135]$. In addition, fabrication of heterostructure with FeSe can also increase its $T_{\mathrm{c}}$, as demonstrated by the observation of very-high- $T_{\mathrm{C}}$ superconductivity in monolayer FeSe films on STO [98]. Therefore fabrication of artificial superlattice based on FeSe is a promising way to obtain high $T_{\mathrm{c}}$. Nabeshima et al. reported on the growth of FeSe/FeTe superlattice films by a PLD technique [136]. Clear satellite peaks in XRD patterns of the grown films indicated periodic stacking structure of FeSe and FeTe. The grown FeSe/FeTe superlattice films on $\mathrm{CaF}_{2}$ and LAO showed $T_{C}$ higher than those of bulk FeSe and FeSe films on $\mathrm{CaF}_{2}$. This is exiting results if the observed enhancement in $T_{\mathrm{C}}$ is due to their superstructure. However interdiffusion of Se and Te was indicated by XRD and TEM observations, and a formation of $\mathrm{FeSe}_{1-x} \mathrm{Te}_{x}$ may result in the enhanced $T_{\mathrm{c}}$. Therefore, suppression of the diffusion of atoms is necessary by improving the growth procedure or by changing FeTe with other materials. Another interesting results in the FeSe/FeTe superlattice films by Nabeshima et al. was that the FeTe layers in the films were coherently strained to the underlying FeSe layers. This demonstrates that introduction of an $\mathrm{FeSe}_{1-x} \mathrm{Te}_{x}$ buffer layer will make it possible to control the lattice strain in an $\mathrm{FeSe}_{1-x} \mathrm{Te}_{x}$ film on it.

To summarize this section, study on the thin film growth of Fe chalcogenides has been carried out as extensively as that for 122-system. FeSe ${ }_{1-x} \mathrm{Te}_{x}$ has been found to have good $J_{\mathrm{c}}$ characteristics especially under high magnetic fields, and is expected to be applied to high-field superconducting magnets. Recently successful suppression of phase separation by the film growth revealed that optimal composition in terms of $T_{\mathrm{C}}$ is in the composition region where bulk crystal cannot be obtained. Therefore, research on the composition dependence of $J_{\mathrm{C}}$ is necessary to clarify the optimal composition for application of these materials, as well as improvement of $J_{c}$ characteristics by introduction of artificial pinning centers. In view of fundamental study, on the other hand, $\mathrm{FeSe}_{1-x} \mathrm{Te}_{x}$ is unique in that 
(i) FeSe shows a structural transition without a magnetic order unlike other iron-based superconductors and in that (ii) $\mathrm{FeSe}_{1-x} \mathrm{Te}_{x}$ is considered to be in the BCS-BEC crossover region because of its very large $\Delta / \epsilon_{\mathrm{F}}\left(\Delta\right.$ and $\epsilon_{\mathrm{F}}$ are the superconducting gap size and the Fermi energy, respectively) [137-139]. Although it has been an obstacle to research on these materials that samples are not available in a wide composition region of $0.1<x<0.4$, successful growth of single crystalline films in a whole composition region will promote progress in the research.

\subsection{1-System $M L$}

The report on the high $T_{\mathrm{C}}$ superconductivity in monolayer FeSe films on $\mathrm{SrTiO}_{3}$ [98] has generated considerable research interest because it may set a new record of $T_{C}$ in iron-based superconductors that has been unchanged since 2008, and because there is a possibility that the $T_{c}$ value exceeds boiling point of liquid nitrogen. Wang et al. observed superconducting-like large energy gaps by scanning tunneling spectroscopy (STS) at low temperatures in monolayer FeSe films on $\mathrm{TiO}_{2}$-terminated STO grown by MBE [98]. They demonstrated a U-shaped gap with magnitude of $40.2 \mathrm{meV}$ at $4.2 \mathrm{~K}$. This means that the size of the superconducting gap, $\Delta$, is $20.1 \mathrm{meV}$, which corresponds to $T_{\mathrm{C}}$ of approximately $80 \mathrm{~K}$ assuming the same gap ratio, $2 \Delta / k_{B} T_{c}$, as that of bulk FeSe. Interestingly, no energy gaps were found in $2 \mathrm{ML}$ FeSe, suggesting this phenomena originates from the interface between FeSe and STO.

Soon after the discovery, angle-resolved photoemission spectroscopy (ARPES) measurements of monolayer FeSe were performed, which revealed that monolayer FeSe on STO has only electron Fermi surfaces at $M$ point, suggesting the samples were heavily electron-doped [13]. Disappearance of the hole Fermi surface in monolayer FeSe is similar to the case of $\mathrm{K}_{x} \mathrm{Fe}_{2-y} \mathrm{Se}_{2}$ [15]. On the other hand, the Fermi surface of films with thickness of equal to or thicker than 2 ML is completely different from that of monolayer films; it consists of both hole and electron-like Fermi surfaces, similar to that of bulk crystals. The origin of the electron doping was found to be charge transfer from the STO substrates [140]. Opening of the energy gap was also confirmed by ARPES studies, which demonstrate that the gap persists up to $\sim 65 \mathrm{~K}$ [13]. The gap structure is nearly isotropic, consistent with the U-shaped spectrum observed by STS measurements. More importantly, a bending back of the electron band after the gap opening was observed, which suggests electron-hole symmetry of the quasiparticles in the superconducting state [141].

Although the best demonstration of superconductivity is observation of zero resistivity and the Meissner effect, there are some difficult problems in these measurements for monolayer FeSe on STO. One is that air exposure destroys the superconductivity. Another is high conductivity of the treated STO substrates. According to Wang et al., the substrates are etched by Se flux at $950{ }^{\circ} \mathrm{C}$ in the growth chamber just before the deposition, which make the substrate conductive [98]. Despite these difficulties, Wang et al., in their first paper, attempted transport measurements using a 5 ML FeSe films capped with amorphous Si without pretreatment of the substrate at high temperature in vacuum, and they obtained $T_{\mathrm{c}}>30 \mathrm{~K}$, much higher than bulk FeSe. Later, ex-situ measurements of transport properties and magnetization of monolayer FeSe on STO, using various capping layers. Zhang et al. prepared monolayer FeSe on insulating STO substrates with $10 \mathrm{ML}$ of FeTe protection layers [142]. The samples shows $T_{\mathrm{c}}^{\text {onset }}=40 \mathrm{~K}, T_{\mathrm{c}}^{\text {zero }}=23.5 \mathrm{~K}$, and $J_{\mathrm{c}}=1.7 \times 10^{6} \mathrm{~A} / \mathrm{cm}^{2}$ at $2 \mathrm{~K}$. The value of $J_{\mathrm{c}}$ is much larger than bulk FeSe and comparable to that of $\mathrm{FeSe}_{1-x} \mathrm{Te}_{x}$ thin films. Deng et al. measured the dc and ac magnetic susceptibility of 1-4 ML FeSe on STO with FeTe protection layers, and reported that the superconductivity persists up to $45 \mathrm{~K}$ [143]. Subsequently, Zhang et al. improved the protection layers and measured the ac magnetic susceptibility of Se(18 nm)/FeSe(2 ML)/ $\mathrm{Fe}_{0.96} \mathrm{Co}_{0.04} \mathrm{Se}(2 \mathrm{ML}) / \mathrm{FeSe}$ (1 ML)/Nb-doped STO, and demonstrated the onset of diamagnetic signal at $65 \mathrm{~K}$ [144]. 
Ge et al. performed in situ transport measurements on monolayer FeSe for the first time [145]. They used four-point probe which was made on the basis of STM, and observed $T_{\mathrm{C}}$ above $100 \mathrm{~K}$ in monolayer FeSe. The reported $T_{\mathrm{c}}$ value is much higher than those reported by ARPES measurements and other ex-situ measurements. This may be because this four-point probe is local probe and successfully avoided step structure of the substrates, which may suppress the superconductivity for atomically thin films. However further confirmation by other research groups is necessary.

There are three possible origins for the enhancement of $T_{\mathrm{c}}$ in monolayer FeSe on STO: (i) tensile strain; (ii) the electron doping from the substrates; and (iii) the electron-phonon coupling to a phonon mode of STO. Firstly, the lattice stain will affect the superconductivity in monolayer FeSe as the case of bulk FeSe. The monolayer FeSe films are coherently strained to the STO substrates, namely their $a$-axis length is $3.90 \AA$ [98]. Peng et al. grew monolayer FeSe on KTO substrate with STO buffer layer, which had larger $a$-axis length of $3.99 \AA$ [146]. ARPES measurements revealed that the film under stronger tensile strain showed larger superconducting gap of $\Delta \sim 19 \mathrm{meV}$ and higher gap-opening temperature of $T_{\mathrm{g}} \sim 70 \mathrm{~K}$ than films on STO substrates $\left(\Delta \sim 15 \mathrm{meV}\right.$ and $\left.T_{\mathrm{g}} \sim 65 \mathrm{~K}\right)$. The enhancement of $T_{\mathrm{c}}$ under tensile strain in monolayer FeSe is contrary to the case of bulk FeSe, and thus, the enhancement of $T_{\mathrm{c}}$ in monolayer FeSe cannot be explained only by the strain effects.

The second factor is carrier-doping from the substrates. ARPES studies revealed that monolayer FeSe on STO is heavily electron-doped and the hole Fermi surface has vanished. The importance of carrier-doping for the enhanced superconductivity is suggested by the fact that the superconductivity is not observed in the second layer of a bilayer FeSe film, where the band structure is similar to that of bulk FeSe. Miyata et al. investigated carrier-doping effects on superconductivity for FeSe multilayer films by ARPES, where electron-doping was achieved by K adsorption after the growth [147]. They found that when electron is doped even 3-ML thick films have enhanced superconducting gaps while it is smaller compared with monolayer FeSe. They also found that the superconducting phase of 3-ML thick films is dome-shaped in a temperature-vs-doping-level phase diagram, suggesting an unconventional mechanism for the superconductivity. Subsequently, the K-adsorption effects were investigated in detail by other several research groups using ARPES [148,149] or STM/STS [150-152], and their common results were that the electron-doping can enhance the superconductivity even for a 50-ML-thick FeSe film [149] and the superconducting gap size of the doped samples becomes larger as the thickness becomes thinner, suggesting an interfacial effect for the enhancement of superconductivity in monolayer FeSe.

The carrier doping effects were also investigated by ex-situ measurements. Shiogai et al. [153], Lei et al. [154] and Hanzawa et al. [155] independently demonstrated enhancement of $T_{\mathrm{c}}^{\text {onset }}$ to above $40 \mathrm{~K}$ by electron doping using an electric double-layer transistor (EDLT) technique. As the gate voltage is changed, a rapid increase of $T_{\mathrm{c}}$ and sign reversal of Hall coefficient are simultaneously observed, suggesting that enhancement of $T_{\mathrm{C}}$ is related to the Lifshitz transition (disappearance of hole Fermi surface) [154]. Shiogai et al. successfully controlled thickness of the grown FeSe films by electrochemical etching using EDLT, and investigated the dependence of $T_{\mathrm{c}}$ on thickness and substrate $[153,156]$. They found that electron-doped FeSe films on both STO and MgO substrates show almost the same $T_{\mathrm{c}}$ of approximately $40 \mathrm{~K}$. These results seem to be inconsistent with the results of the in situ measurements, which may be due to differences in condition of pretreatment for substrates and post-annealing.

Coupling of electrons in FeSe to phonon of STO substrates has also been considered to be one of the origin of the superconductivity in monolayer FeSe [157]. Lee et al. in their ARPES studies observed a band-dispersion-like structure with the similar shape as the true band dispersions at $\sim 100 \mathrm{meV}$ lower than the true ones (replica bands) [158]. The energy value of $100 \mathrm{meV}$ corresponds to that of a phonon mode of STO substrates, and observation of replica bands suggests strong electron-phonon coupling. They estimated the electron-phonon coupling constant, $\lambda$, to be 0.5 from the relative intensity of replica bands. Large electron-phonon coupling to STO substrates was also suggested by some other experimental results $[151,159,160]$. However, at present, there are no experimental results that 
demonstrate the direct relation between the electron-phonon coupling and the enhancement of the superconductivity. Nevertheless, the interface superconductivity has also been found in related materials such as FeSe on $\mathrm{BaTiO}_{3}$ [161], Te-doped FeSe on STO [162], $\mathrm{KFe}_{2} \mathrm{Se}_{2}$ on STO [148], FeSe on STO(110) [163,164], and FeSe on anatase $\mathrm{TiO}_{2}$ [165]. In addition, replica bands were also observed in monolayer FeSe on anatase $\mathrm{TiO}_{2}$ [166]. The mechanism for the enhancement of superconductivity is considered to be the same among these materials, and thus, comprehensive study including these materials will enrich understanding of the interface superconductivity in FeSe on STO.

To summarize this section, monolayer FeSe on STO is a potential superconductor with much better superconducting properties than other iron-based superconductors. However, the superconducting properties of monolayer FeSe observed by ex-situ measurements are not good compared with other iron-based materials at present. Therefore, there are still many challenges for application of these materials such as improvement of protection layers and fabrication of superlattice utilizing interface effects between FeSe and STO.

On the other hand, in view of fundamental study there has been much progress in understandings of the superconductivity in monolayer FeSe. Interface superconductivity was first proposed by Ginzburg in 1964 [167]. Monolayer FeSe on STO may be the first that demonstrates enhancement of $T_{\mathrm{c}}$ by interface effects. Indeed, in STS and ARPES studies, the thickness dependence of the superconducting gap size of carrier-doped FeSe monolayer films suggests interface effects. Several results such as the observation of replica band by ARPES measurements indicated the strong coupling of FeSe electrons with phonon of STO, and the electron phonon coupling is considered to be the most likely candidate for the interface-enhancement mechanism of the superconductivity. However, the results of ex-situ measurements such as by a EDLT technique are inconsistent with other results of in situ probes such as ARPES and STS. Thus, there is still room for discussion about interface effects on the superconductivity in monolayer FeSe on STO. In particular, successful in situ measurements of electrical resistivity have been reported by only one group, and therefore further confirmation by other groups is necessary.

\section{Substrate Materials in the Films Fabrication of Iron-Based Superconductors}

The substrate material is one of the key parameters for the thin film fabrication. The specifications for substrate materials which are used in the film growth of iron-based superconductors are summarized in Table 1. Generally, the substrate material whose in-plane constant is close to that of the target material is suitable for the epitaxial thin film growth because the lattice mismatch between the film and the substrate is small. Therefore, it seems to be natural that there are no common "optimal" substrate for thin film growth of 1111,122, and 11 since there are large differences in the in-plane lattice constants of these three systems. However, in thin films of 1111,122, and 11, the highest $T_{\mathrm{c}}$ is commonly obtained in the case of $\mathrm{CaF}_{2}$ substrates. Tsukada et al. firstly reported the effectiveness of the $\mathrm{CaF}_{2}$ substrate in the 11 film fabrication [40], and then the fabrication of the film with high $T_{\mathrm{c}}$ were reported in other systems $[31,41,63]$. Here, we define the lattice misfit,

$$
M=100 \times \frac{a_{\text {sub }}-a_{\text {bulk }}}{a_{\text {sub }}},
$$

where $a_{\mathrm{sub}}, a_{\mathrm{bulk}}$ are in-plane lattice constants of substrate and bulk materials (in some substrate materials, the in-plane lattice constant divided by $\sqrt{2}$ is used as $a_{\text {sub }}$ ), respectively. We use the values reported in refs. $[27,63,168,169]$ as $a_{\text {bulk }}$, and the values of $a_{\text {sub }}$, which are summarized in Table 1 . In Figure 1, we plot the lattice constants and $T_{\mathrm{c}}$ for films as a function of $M$. In a simple way, the positive (negative) $M$ is expected to result in the tensile (compressive) strain. From the Poisson effect, the changes of lattice parameters are expected as follows, 


$$
\begin{aligned}
& M>0 \rightarrow a_{\text {film }}>a_{\text {bulk }}, c_{\text {film }}<c_{\text {bulk }} \\
& M<0 \rightarrow a_{\text {film }}<a_{\text {bulk }}, c_{\text {film }}>c_{\text {bulk }}
\end{aligned}
$$

where $a_{\text {bulk }}\left(c_{\text {bulk }}\right)$ and $a_{\text {film }}\left(c_{\text {film }}\right)$ are the $a(c)$-axis lengths of bulk and film, respectively. However, the behaviors shown in Figure 1 are different from these simple expectations, and there is no definite correlation between the lattice misfit and lattice parameters or $T_{\mathrm{c}}$. In 1111 and 122, the in-plane lattice parameters of films on most of substrates are smaller than those of bulk samples. On the other hand, in $11, a_{\text {film }}$ is larger than $a_{\text {bulk }}$ for most of films except for $\mathrm{LaAlO}_{3}$ and $\mathrm{CaF}_{2}$. An important thing is that the films of all these three systems on $\mathrm{CaF}_{2}$ substrates commonly have small in-plane lattice constants and show superconductivity with high $T_{\mathrm{c}}$. Additionally, it is noteworthy that these commonly-observed compressive strains can be expected in 1111 and 122 but cannot be expected in 11 from the values of $M$. Why do films of iron-based superconductors on $\mathrm{CaF}_{2}$ substrates receive the compressive strains? One possible reason may be a thermal expansion. In the case of large coefficient of thermal expansion, it is possible that the size mismatch is relaxed at the deposition temperature or that the large compressive strain is induced in the cooling process from the deposition temperature to room temperature. In Figure 2, we plot the lattice constants and $T_{\mathrm{c}}$ for films as a function of coefficient of thermal expansion at $300 \mathrm{~K}, \alpha_{300 \mathrm{~K}} \cdot T_{\mathrm{c}}$ and $c_{\text {film }}\left(a_{\text {film }}\right)$ roughly increase(decrease) with increasing $\alpha_{300 \mathrm{~K}}$, while there are a few exception, for example $\mathrm{BaF}_{2}$ and $\mathrm{SrF}_{2}$ substrates. The changes of the lattice parameters in Figure 2 suggest that large $\alpha_{300 K}$ induces the strong compressive strain, which results in the enhancement of $T_{\mathrm{c}}$. The reason why the behaviors in the $\mathrm{BaF}_{2}$ and $\mathrm{SrF}_{2}$ substrates with large $\alpha_{300 \mathrm{~K}}$ are different from the above expectations may be that the lattice mismatches in these two substrates are too large.

Table 1. In-plane lattice parameters, $a_{\mathrm{sub}}$, and coefficients of thermal expansion at $300 \mathrm{~K}, \alpha_{300 \mathrm{~K}}$, for substrate materials.

\begin{tabular}{ccc}
\hline Substrate Material & $\boldsymbol{a}_{\text {sub }}(\AA)$ & $\boldsymbol{\alpha}_{\mathbf{3 0 0 K}}\left(\times \mathbf{1 0}^{-\mathbf{6}} 1 / \mathrm{K}\right)$ \\
\hline Yttria stabilized zirconia $\left(\mathrm{Y}: \mathrm{ZrO}_{2}\right)$ & $3.635(a / \sqrt{2})[170]$ & $11[171]$ \\
$\mathrm{YAlO}_{3}(\mathrm{YAO})$ & $3.716(a / \sqrt{2})[172]$ & $3.8[173]$ \\
$\mathrm{LaSrAlO}_{4}(\mathrm{LSAO})$ & $3.754[174]$ & $7.55[175]$ \\
$\mathrm{LaAlO}_{3}(\mathrm{LAO})$ & $3.793(a / \sqrt{2})[176]$ & $5.77[177]$ \\
$\mathrm{LaSrGaO}_{4}(\mathrm{LSGO})$ & $3.844[178]$ & $10.05[179]$ \\
$(\mathrm{La}, \mathrm{Sr})(\mathrm{Al}, \mathrm{Ta}) \mathrm{O}_{3}(\mathrm{LSAT})$ & $3.869(a / \sqrt{2})[180]$ & $8.22[177]$ \\
$\mathrm{SrTiO}_{3}(\mathrm{STO})$ & $3.905[181]$ & $10.3[182]$ \\
$\mathrm{MgO}_{\mathrm{CaF}}$ & $4.211[183]$ & $10.5[182]$ \\
$\mathrm{SrF}_{2}$ & $3.865(a / \sqrt{2})[184]$ & $18.9[185]$ \\
$\mathrm{BaF}_{2}$ & $4.101(a / \sqrt{2})[186]$ & $18.1[185]$ \\
$\mathrm{GaAs}$ & $4.382(a / \sqrt{2})[187]$ & $18.4[185]$ \\
\hline
\end{tabular}



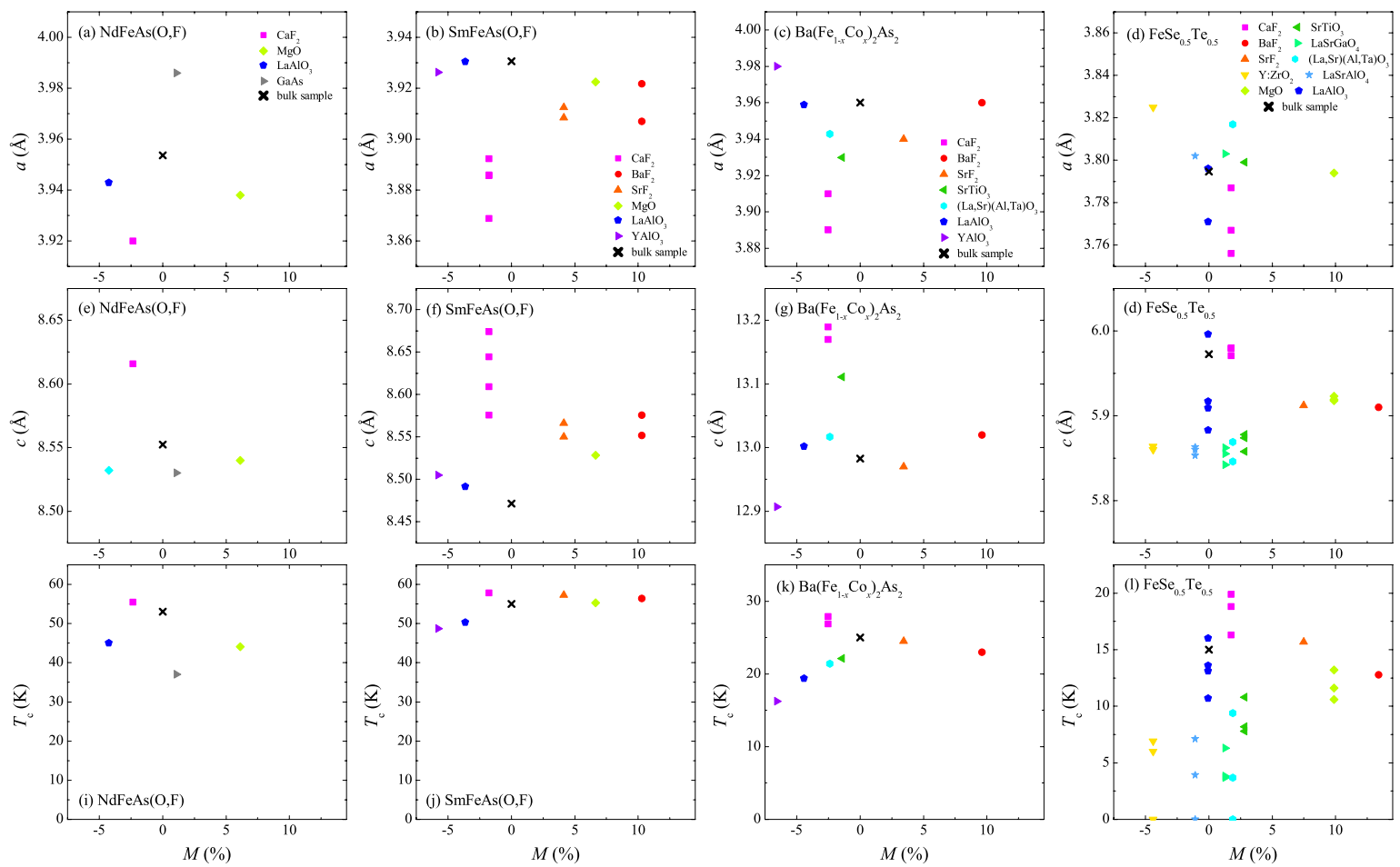

Figure 1. Dependence of lattice parameters and $T_{\mathrm{c}}$ for $\mathrm{NdFeAs}(\mathrm{O}, \mathrm{F})$ films [189], $\operatorname{SmFeAs}(\mathrm{O}, \mathrm{F})$ films [190], $\mathrm{Ba}\left(\mathrm{Fe}_{1-x} \mathrm{Co}_{x}\right)_{2} \mathrm{As}_{2}$ films [51,63], and $\mathrm{FeSe}_{0.5} \mathrm{Te}_{0.5}$ films [40,52,191] on lattice misfit, $M$.
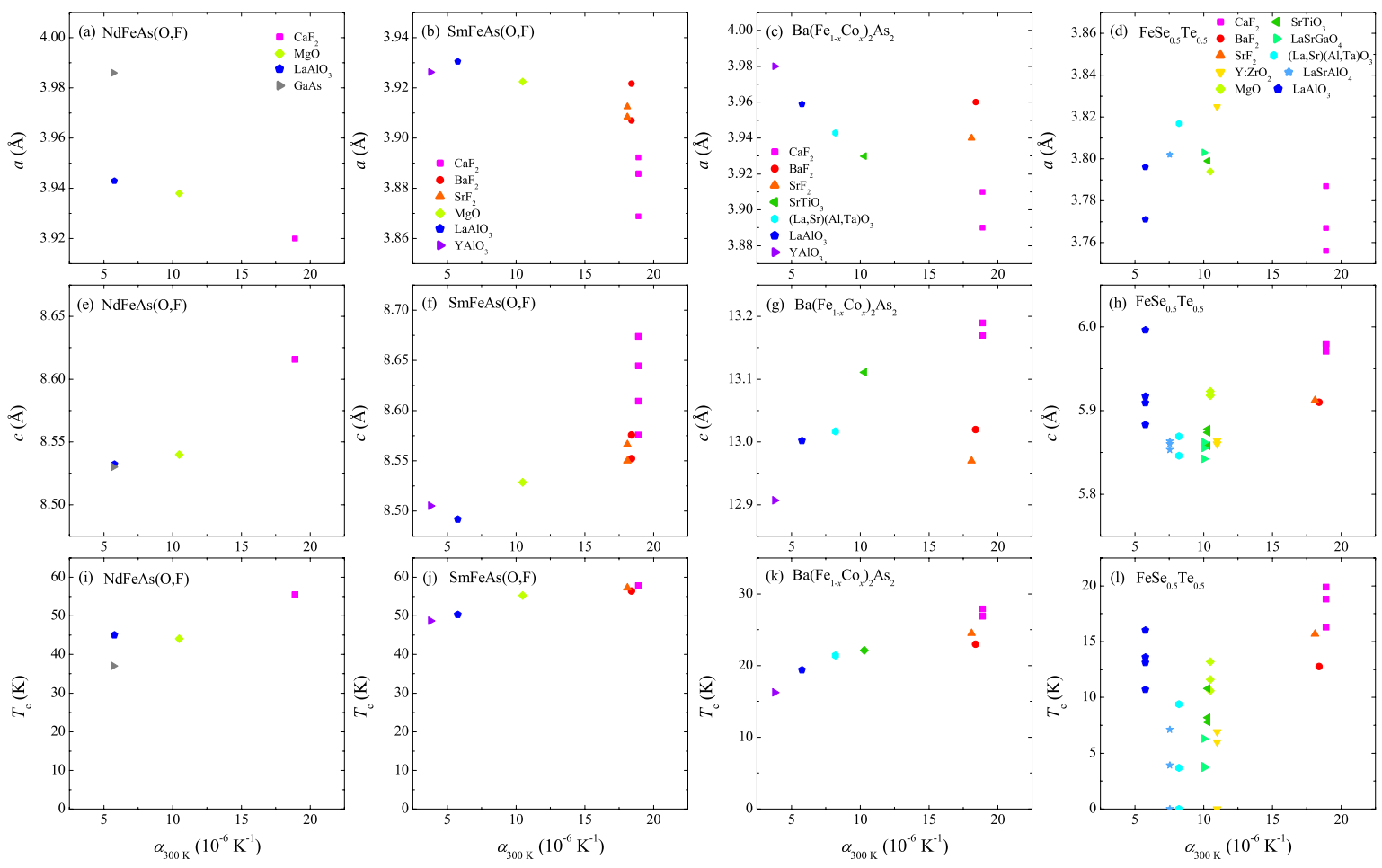

Figure 2. Dependence of lattice parameters and $T_{\mathrm{c}}$ for $\mathrm{NdFeAs}(\mathrm{O}, \mathrm{F})$ films [189], $\operatorname{SmFeAs}(\mathrm{O}, \mathrm{F})$ films [190], $\mathrm{Ba}\left(\mathrm{Fe}_{1-x} \mathrm{Co}_{x}\right)_{2} \mathrm{As}_{2}$ films [51,63], and $\mathrm{FeSe}_{0.5} \mathrm{Te}_{0.5}$ films [40,52,191] on the coefficient of thermal expansion at $300 \mathrm{~K}, \alpha_{300 \mathrm{~K}}$.

While the large value of $\alpha_{300 \mathrm{~K}}$ is important for the compressive strain and high $T_{\mathrm{c}}$ in films of iron-based superconductors on $\mathrm{CaF}_{2}$ substrates, all properties for films on $\mathrm{CaF}_{2}$ cannot be explained 
only by the thermal expansion and there seem to be particular things in each materials. In 1111, $M$ is the closest to zero among three systems, which is slightly negative. Indeed, $a_{\text {film }}$ of Sm1111 films on $\mathrm{CaF}_{2}$ is $\sim 3.87-3.89 \AA$ which is smaller than the $a$-axis length of bulk samples and is close to $a_{\text {sub }} \sim 3.865 \AA$ for $\mathrm{CaF}_{2}$ in Table 1 . From the measurement of the cross-sectional transmission electron microscopy (TEM), the interface between the film and the substrate is smooth and the atomic ordering across the interface is nearly perfect in Sm1111 films on $\mathrm{CaF}_{2}$ [46,192]. Therefore, $\mathrm{CaF}_{2}$ is an appropriate substrate material for this system also in terms of the lattice mismatch. In addition, the $\mathrm{F}$ diffusion into the film from the $\mathrm{CaF}_{2}$ substrate was confirmed [46] and the $\mathrm{CaF}_{2}$ substrate plays a role as the F dopant [31,41]. Therefore, in terms of both the lattice mismatch and the F dopant, the $\mathrm{CaF}_{2}$ is suitable as a substrate material for the film growth of 1111 . In 122, the in-plane lattice constant of $\mathrm{CaF}_{2}$ is slightly smaller than that of $\mathrm{BaFe}_{2-x} \mathrm{Co}_{x} \mathrm{As}_{2}$ bulk sample, which is expected to result in the compressive strain. Indeed, $\mathrm{BaFe}_{2-x} \mathrm{Co}_{x} \mathrm{As}_{2}$ films on $\mathrm{CaF}_{2}$ substrates receive the compressive strain, which is similar to the behaviors observed in 1111 films. However, there is a clear difference in the micro-structure near the interface between the film and the substrate between 1111 and 122 films on $\mathrm{CaF}_{2}[46,63,192]$. In the case of $\mathrm{BaFe}_{2-x} \mathrm{Co}_{x} \mathrm{As}_{2}$ films on $\mathrm{CaF}_{2}$, the interface between the film and the substrate is unclear and the reactive layers exist just on the $\mathrm{CaF}_{2}$ substrate. These layers contain some Ba deficiencies, which are unstable and are easy to change into the amorphous-like layers after aging [46]. The energy dispersive $\mathrm{X}$-ray analysis (EDX) revealed that a small amount of Ba and Ca was inter-diffused at the interface. In $\mathrm{BaFe}_{2-x} \mathrm{Co}_{x} \mathrm{As}_{2}$ films on oxide substrates, the similar diffusion of $\mathrm{Ba}$ from the film to the substrate was also reported by Haindl et al. [24]. Therefore, the $a$-axis length was not reduced to the length of the $\mathrm{CaF}_{2}$ substrate because the initial 122 layer deformed by the underlying $\mathrm{CaF}_{2}$ with a Ba-partially-substituted $\mathrm{CaF}_{2}$. Finally, the in-plane lattice constant of $\mathrm{FeSe}_{0.5} \mathrm{Te}_{0.5}$ film on the $\mathrm{CaF}_{2}$ substrate is smaller than those of $\mathrm{FeSe}_{0.5} \mathrm{Te}_{0.5}$ and FeSe bulk samples, although $M$ is positive in 11. Thus, the unexpected in-plane shrinkage of the $\mathrm{FeSe}_{0.5} \mathrm{Te}_{0.5}$ film cannot be explained neither by the simple lattice mismatch nor by the deviations in Te content. Ichinose et al. insisted the F diffusion into film from the $\mathrm{CaF}_{2}$ substrate [45] and/or the formation of the Se-deficient layer [101] as the origin of the nontrivial compressive strain. In the cross-sectional TEM image, a reactive region exists at the interface between the film and the substrate also in $\mathrm{FeSe}_{0.5} \mathrm{Te}_{0.5}$ film on the $\mathrm{CaF}_{2}[45,46]$. Although this seems to be similar to the initial layer of $\mathrm{BaFe}_{2-x} \mathrm{Co}_{x} \mathrm{As}_{2}$ film with some Ba deficiencies, these are quite different and the reactive layer in $\mathrm{FeSe}_{0.5} \mathrm{Te}_{0.5}$ film exist not at the initial layer of the film but at the top of the $\mathrm{CaF}_{2}$ substrate. The energy dispersive $\mathrm{X}$-ray analysis (EDX) revealed that a small amount of the interdiffusion of $\mathrm{F}$ and $\mathrm{Se}$ at the interface, and the reactive layer at the interface is considered to play a role in the compressive strain as a buffer layer.

In the film fabrication of iron-based superconductors, the material with a large coefficient of thermal expansion is considered to be suitable as the substrate for obtaining films of iron-based superconductors with high $T_{\mathrm{c}} \cdot \mathrm{CaF}_{2}$ has a large coefficient of thermal expansion and a relatively small value of lattice misfit, and thus is an appropriate substrate material for the film fabrication of iron-based superconductors.

\section{Comparison in Phase Diagram between Bulk Single Crystal and Epitaxial Thin Films in 11 System}

The progress of thin film growth technique enables us to investigate a systematic change in physical properties with the composition in the thin film and to compare the phase diagram between the bulk single crystal and the epitaxial thin film [131,193]. Here, we introduce the whole phase diagram in $\mathrm{FeSe}_{1-x} \mathrm{Te}_{x}$ thin films.

The iron-chalcogenide superconductor, FeSe $\left(T_{\mathrm{c}} \approx 8 \mathrm{~K}\right)$ [18], initially attracted attention because it has the simplest crystal structure among iron-based superconductors and its $T_{\mathrm{C}}$ strongly depends on applied pressures [194-196]. FeSe undergoes a structural transition from tetragonal to orthorhombic at a structural transition temperature, $T_{\mathrm{s}} \approx 90 \mathrm{~K}$ and does not show any long-range magnetic orders at ambient pressure. This is quite contrast to other iron-based superconductors, where a structural 
and a magnetic phase transition occur almost simultaneously. Recently, this unique feature of FeSe has attracted lots of attention for exploring the relation between superconductivity and a structural and/or nematic order. Since establishment of the method of growth of FeSe single crystals with high quality [197], many studies on this material have been performed. For investigating this system, it is quite natural to become interested in the substitution effect, for example FeSe $1-x S_{x}$ and $\mathrm{FeSe}_{1-x} \mathrm{Te}_{x}$. The reported results in $\mathrm{FeSe}_{1-x} \mathrm{Te}_{x}$ bulk samples [27,198-202] are summarized in Figure 3a. FeSe shows a structural/nematic transition at $T_{\mathrm{S}} \sim 90 \mathrm{~K}$ [199]. While the structural transition is still observed at $x \approx 0.43$ [202], it disappears at $x \approx 0.5$ where the highest $T_{\mathrm{C}}$ of $15 \mathrm{~K} \mathrm{in} \mathrm{FeSe}_{1-x} \mathrm{Te}_{x}$ is obtained [200]. The values of $T_{\mathrm{C}}$ do not change at $x \approx 0.5-0.8$ very much [198]. Additionally, the end member material FeTe opposite to FeSe shows a structural transition from tetragonal to monoclinic at $T_{\mathrm{S}}\left(T_{\mathrm{N}}\right) \sim 70 \mathrm{~K}$ and simultaneously develops a bicollinear antiferromagnetic (AF) order [200,203] whose AF magnetic structure is different from those of other iron-based superconductors [29,204]. In the Te-substituted materials, it is well-known that a phase separation occurs at $0.1 \leq x \leq 0.4$ and that the single phase sample with these compositions are not available [27]. This is a large problem for investigating the Te substitution effect in FeSe. Recently, we succeeded in obtaining $\mathrm{FeSe}_{1-x} \mathrm{Te}_{x}$ thin films with these compositions by a pulsed laser deposition (PLD) method [131]. Zhuang et al. also reported the successful suppression of phase separation by using film technique around the same time, independently [130]. Generally, the film deposition, whose process involves crystal growth in a thermodynamically non-equilibrium state, provides an avenue for the synthesis of a material with a metastable phase. The suppression of phase separation is probably brought about by this feature of film fabrication process. The temperature dependence of resistivity of $\mathrm{FeSe}_{1-x} \mathrm{Te}_{x}$ thin films on $\mathrm{CaF}_{2}(x=0-1)$ and $\mathrm{LaAlO}_{3}(x=0-0.7)$ substrates is summarized in Figure 4. A superconducting transition is observed in all films except for FeTe films on $\mathrm{CaF}_{2}$. The optimum Te content for obtaining the highest $T_{\mathrm{c}}, x_{\mathrm{opt}}$, depends on a kind of substrate. The highest $T_{\mathrm{c}}$ is $\sim 23 \mathrm{~K}$ at $x_{\mathrm{opt}}=0.2$ for $\mathrm{CaF}_{2}$ and $\sim 19 \mathrm{~K}$ at $x_{\mathrm{opt}}=0.4$ for LAO. These values of $T_{\mathrm{C}}$ are larger than the highest $T_{\mathrm{C}} \sim 15 \mathrm{~K}$ [27] observed in $\mathrm{FeSe}_{1-x} \mathrm{Te}_{x}$ bulk samples. It is noteworthy that the highest $T_{\mathrm{c}}$ was obtained at or around the phase-separation region for films on both substrates. This enhancement of $T_{\mathrm{C}}$ seems to be consistent with a recent theoretical study which concludes that a superconducting phase with high $T_{\mathrm{C}}$ is placed around a electronic phase separation region [205]. Looking at the normal-state behavior, the residual resistivity ratio, $R R R(=\rho(300 \mathrm{~K}) / \rho(20 \mathrm{~K}))$, of these films decreases with increasing $x$. Note that there is a kink around $90 \mathrm{~K}$ in the $\rho-T$ curve for annealed FeSe film on $\mathrm{LaAlO}_{3}$ with large value of $R R R \approx 7$. This behavior is familiar in FeSe bulk single crystals, and is due to the structural transition from tetragonal to orthorhombic $[197,199]$. A structural/nematic transition temperature, $T_{\mathrm{s}}$, for $\mathrm{FeSe}_{1-x} \mathrm{Te}_{x}$ films can be estimated from the temperature dependence of the temperature derivative of resistivity shown in Figure $5 \mathrm{~b}$ as FeSe bulk samples. $T_{\mathrm{S}}$ is considered to be a temperature where a temperature derivative of resistivity takes its local minimum (maximum) for FeSe single crystals with large values of $R R R$ [197] (samples with small values of $R R R$ [206,207]). We can define $T_{\mathrm{s}}$ at $x=0-0.1$ for films on $\mathrm{CaF}_{2}$ and $x=0-0.3$ for films on LAO [133]. At the other compositions, there are no peak- nor dip- structure in a temperature derivative of resistivity, which shows that structural/nematic transition does not occur. To understand the relation between $T_{\mathrm{S}}$ and $T_{\mathrm{C}}$, these values are plotted as a function of $x$ in Figure $3 b$. In this figure, the horizontal axis is the nominal Te content of the polycrystalline target. We strongly believe that Te contents of the grown films are nearly identical to the nominal values of the targets, because the $c$-axis lengths of the films exhibit almost linear dependence on $x$ including two end member materials, FeSe and FeTe [131,133]. Therefore, in Figure 3b, the horizontal axis is able to be regarded as the Te content of the resultant film. In films on both substrates, $T_{\mathrm{S}}$ decreases with increasing $x$, and then, at $x=x_{\mathrm{opt}}$, the structural/nematic transition is completely suppressed and the highest $T_{\mathrm{C}}$ is realized at the same time. Note that $T_{\mathrm{S}}$ is lower for an FeSe film on $\mathrm{CaF}_{2}$ than that for LAO. This is due to the in-plane lattice strain. Indeed, a recent ARPES measurement for strained FeSe films revealed that the nematicity weakens as the $a$-axis length of the films shrinks [208]. 

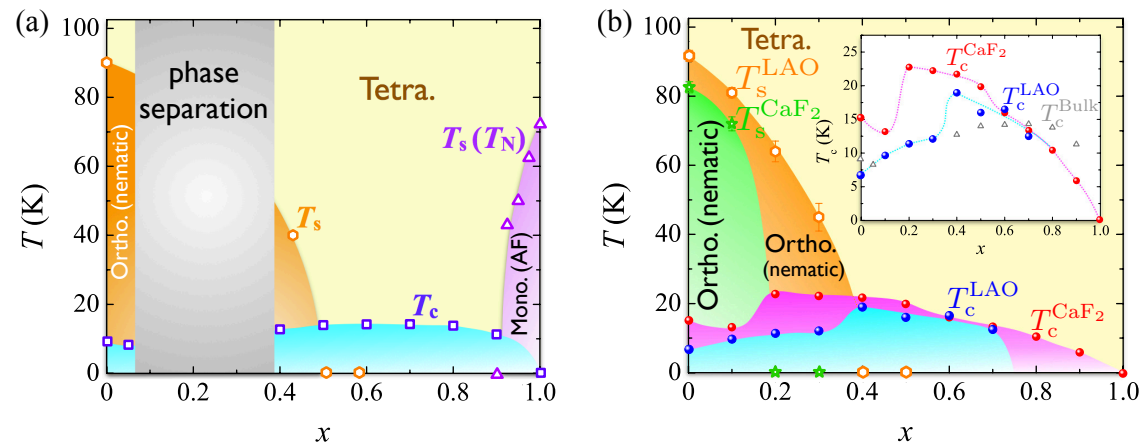

Figure 3. Phase diagrams in (a) bulk samples [27,198-202] and (b) films of $\mathrm{FeSe}_{1-x} \mathrm{Te}_{x}$. Blue circles (red circles) and orange hexagons (light-green stars) represent $T_{\mathrm{C}}^{\text {onset }}$ and $T_{S}$ of the $\mathrm{FeSe}_{1-x} \mathrm{Te}_{x}$ thin films fabricated on LAO [133] $\left(\mathrm{CaF}_{2}[131,132,209]\right)$, respectively. In the inset, the dependence of $T_{\mathrm{C}}^{\text {onset }}$ on Te content $x$ is shown. The values of $T_{\mathrm{C}}$ for bulk samples estimated from the magnetic susceptibility measurements are shown as gray triangles $[27,198]$. The dashed curves in the inset are guides for the eye. The words of "ortho.", "tetra.", "mono.", and "AF" are abbreviations for orthorhombic, tetragonal, monoclinic phases, and bicollinear antiferromagnetic-ordered state, respectively.
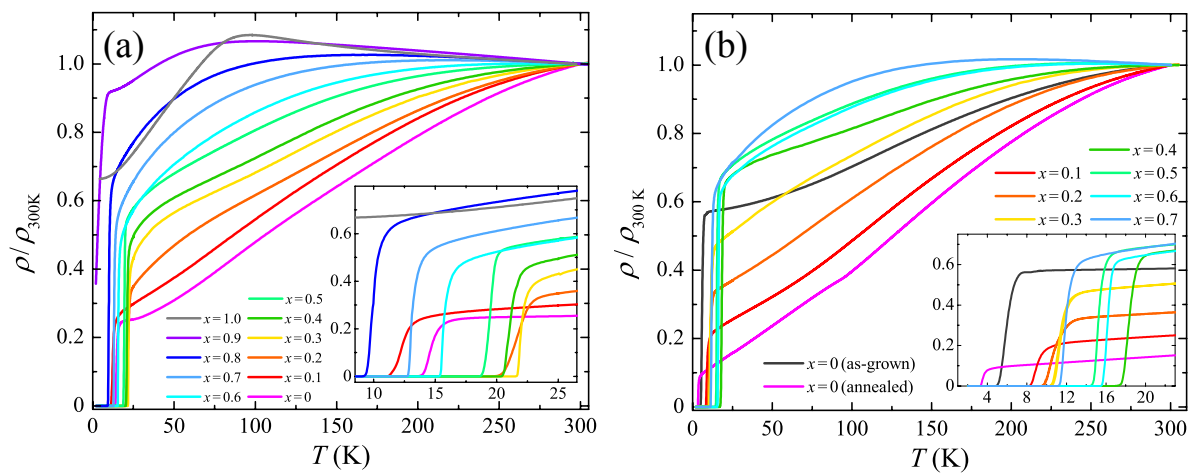

Figure 4. Temperature dependence of resistivity, $\rho$, for $\mathrm{FeSe}_{1-x} \mathrm{Te}_{x}$ films on (a) $\mathrm{CaF}_{2}$ and (b) $\mathrm{LaAlO}_{3}$ substrates.
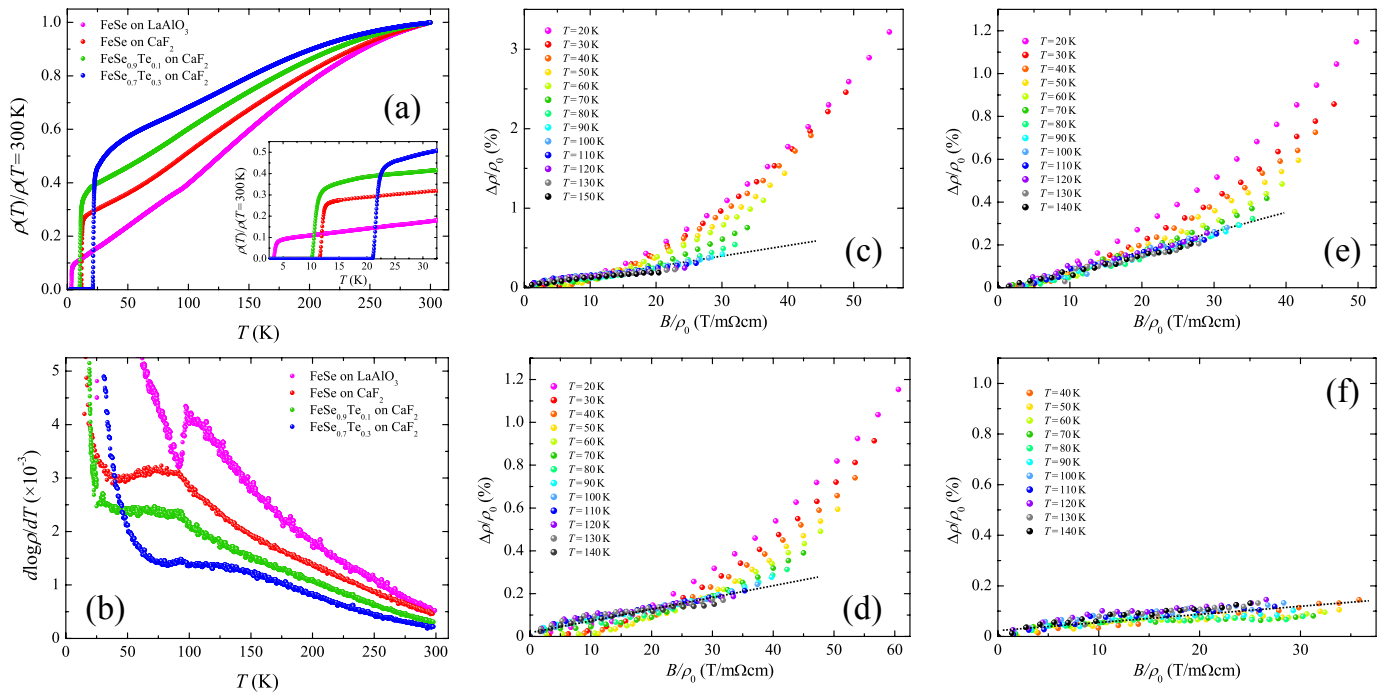

Figure 5. Temperature dependence of (a) resistivity, $\rho$; and (b) $d \log \rho / d T$ for FeSe film on $\mathrm{LaAlO}_{3}$ and $\mathrm{FeSe}_{1-x} \mathrm{Te}_{x}$ films on $\mathrm{CaF}_{2}$ with $x=0,0.1,0.3$; Kohler Plot of (c) FeSe film on $\mathrm{LaAlO}_{3}$ and $\mathrm{FeSe}_{1-x} \mathrm{Te}_{x}$ films on $\mathrm{CaF}_{2}$ with (d) $x=0$; (e) $x=0.1$; and (f) $x=0.3$. 
Here, for discussing the relation between local structural parameters and superconductivity, we focus on the peak intensity of out-of-plane XRD patterns [131,133], especially the intensity ratio of the 003 reflections to the 002 reflections, $r=I_{003} / I_{002}$, where $I_{002}$ and $I_{003}$ are intensities of 002 and 003 reflections, respectively. The structural analysis of $\mathrm{FeSe}_{1-x} \mathrm{Te}_{x}$ bulk samples at room temperatures revealed that the space group and the atomic coordinates other than the $z$-coordinate of the Chalcogen (Se or Te) site did not change with changing Te contents [210]. Thus, from these results in bulk samples [210], we assume (1) the space group is $P 4 / n m m$ (no. 129); (2) only the $z$-coordinate of the Chalcogen (Se or Te) site, $z_{C h}$, depends on $x$ and the other atomic coordinates are constant with $x$; (3) occupancies at each atomic site are the same as the chemical compositions, and simulate XRD patterns of $\mathrm{FeSe}_{1-x} \mathrm{Te}_{x}$ by using VESTA and RIETAN programs [211]. The parameters used in these simulations are summarized in Table 2. We confirmed that the values of $r$ strongly depend on $z_{C h}$. In $\mathrm{FeSe}_{0.5} \mathrm{Te}_{0.5}$ films [92], the similar arguments have been provided by Bellingeri et al. We estimate $z_{C h}$ to reproduce the experimentally-obtained intensity ratio of the 003 reflections to the 002 reflections, $r_{\exp }=I_{003}^{\exp } / I_{002}^{\exp }$. By using the obtained values of $z_{C h}$ and the lattice constants of $a$ and $c$, we calculate the chalcogen height from the iron plane, $h$, and the bond angle, $\alpha$, whose definitions are shown in Figure 6, by using the relation,

$$
h=c \cdot z_{C h}=\frac{a}{2 \tan \frac{\alpha}{2}} .
$$

the results of these calculations are summarized in Table 3. The structural parameters, $\alpha$ and $h$, are well-accepted to be critical ones to determine values of $T_{\mathrm{c}}[95,96,212]$, and the optimum values are empirically known to be $109.48 \mathrm{deg}$. for $\alpha$ [95,96] and $1.38 \AA$ for $h$ [212]. Figure 7 is the relation between $\alpha$ or $h$ and the Te content, $x$. In these figures, we set error bars for the data of $\alpha$ and $h$ by considering the influences of the off-stoichiometry and the differences of $r_{\exp }$ depending on samples. Both parameters get close to their optimum values with decreasing $x$, which probably results in the increase of $T_{\mathrm{c}}$ at $x>x_{\mathrm{opt}}$ as shown in Figure 3b. We plot values of $T_{\mathrm{c}}$ as a function of $\alpha$ or $h$ in Figure 8. Focussing on the data of samples without the structural transition (closed symbols), the values of $T_{\mathrm{c}}$ increase when these parameters get close to their optimum values. This indicates that the well-known empirical laws between $T_{\mathrm{c}}$ and $\alpha$ or $h[95,96,212]$ can be applicable to 11 materials, and the increase of $T_{\mathrm{c}}$ at $x \geq x_{\mathrm{opt}}$ can be explained by the optimization of these structural parameters. On the other hand, the samples with the structural transition, which are represented by open symbols in Figure 8, show rather low $T_{\mathrm{c}}$ despite that their $\alpha$ and $h$ are very close to their optimum values. These results confirmed that the suppression of $T_{\mathrm{c}}$ in films with $x<x_{\mathrm{opt}}$ cannot be explained by the local structural parameters. Therefore, we believe that the sudden change in $T_{\mathrm{c}}$ at $x_{\mathrm{opt}}$ in Figure $3 \mathrm{~b}$ is probably related to the structural/nematic transition since the Te content where $T_{\mathrm{S}}$ disappears coincides $x_{\mathrm{opt}}$ [133], and that the orthorhombic distortion and/or nematic order may not be favorable for the superconductivity in iron chalcogenides.

Table 2. Atomic coordinates and occupancies in the XRD simulation.

\begin{tabular}{ccccc}
\hline Atom & $\boldsymbol{x}_{\text {atom }}$ & y atom $_{\text {atom }}$ & $z_{\text {atom }}$ & Occupancy \\
\hline $\mathrm{Fe}$ & 0.75 & 0.25 & 0 & 1 \\
$\mathrm{Se} / \mathrm{Te}$ & 0.25 & 0.25 & $z_{C h}$ & $1-x / x$ \\
\hline
\end{tabular}



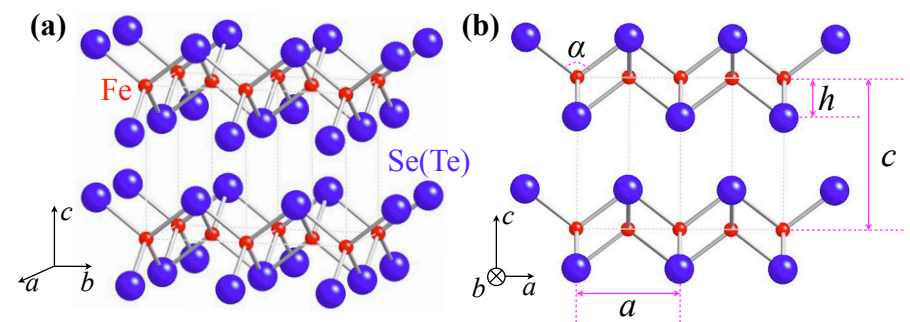

Figure 6. (a) Schematic crystal structure of $\mathrm{FeSe}_{1-x} \mathrm{Te}_{x}$; (b) Front view of $\mathrm{FeSe}_{1-x} \mathrm{Te}_{x}$.

Table 3. Summary of the estimation for structural parameters in $\mathrm{FeSe}_{1-x} \mathrm{Te}_{x}$ films by the XRD simulation.

\begin{tabular}{cccccccc}
\hline $\boldsymbol{x}$ & Substrate & $\boldsymbol{a}(\AA)$ & $\boldsymbol{c}(\AA)$ & $\boldsymbol{r}$ exp & $z_{C h}$ & $\boldsymbol{\alpha}($ deg. $)$ & $\boldsymbol{h}(\AA)$ \\
\hline 0 & $\mathrm{CaF}_{2}$ & 3.735 & 5.584 & 2.410 & 0.239 & 108.80 & 1.337 \\
0.1 & $\mathrm{CaF}_{2}$ & 3.756 & 5.632 & 1.665 & 0.240 & 108.61 & 1.349 \\
0.2 & $\mathrm{CaF}_{2}$ & 3.722 & 5.755 & 2.215 & 0.249 & 104.80 & 1.433 \\
0.3 & $\mathrm{CaF}_{2}$ & 3.748 & 5.869 & 1.592 & 0.248 & 104.35 & 1.455 \\
0.4 & $\mathrm{CaF}_{2}$ & 3.758 & 5.758 & 1.733 & 0.253 & 103.33 & 1.486 \\
0.5 & $\mathrm{CaF}_{2}$ & 3.771 & 5.996 & 1.483 & 0.254 & 102.24 & 1.520 \\
0.6 & $\mathrm{CaF}_{2}$ & 3.752 & 6.066 & 1.477 & 0.256 & 100.70 & 1.555 \\
0.7 & $\mathrm{CaF}_{2}$ & 3.755 & 6.132 & 1.143 & 0.255 & 100.47 & 1.562 \\
0 & $\mathrm{LaAlO}_{3}$ & 3.750 & 5.530 & 1.137 & 0.228 & 112.10 & 1.262 \\
0.1 & $\mathrm{LaAlO}_{3}$ & 3.779 & 5.558 & 2.383 & 0.245 & 108.46 & 1.361 \\
0.3 & $\mathrm{LaAlO}_{3}$ & 3.776 & 5.815 & 2.658 & 0.256 & 103.39 & 1.491 \\
0.4 & $\mathrm{LaAlO}_{3}$ & 3.734 & 5.883 & 1.848 & 0.254 & 102.66 & 1.494 \\
0.5 & $\mathrm{LaAlO}_{3}$ & 3.789 & 5.977 & 1.748 & 0.256 & 102.10 & 1.531 \\
0.6 & $\mathrm{LaAlO}_{3}$ & 3.770 & 6.042 & 1.267 & 0.254 & 101.71 & 1.534 \\
0.7 & $\mathrm{LaAlO}_{3}$ & 3.797 & 6.126 & 1.201 & 0.256 & 100.98 & 1.565 \\
\hline
\end{tabular}
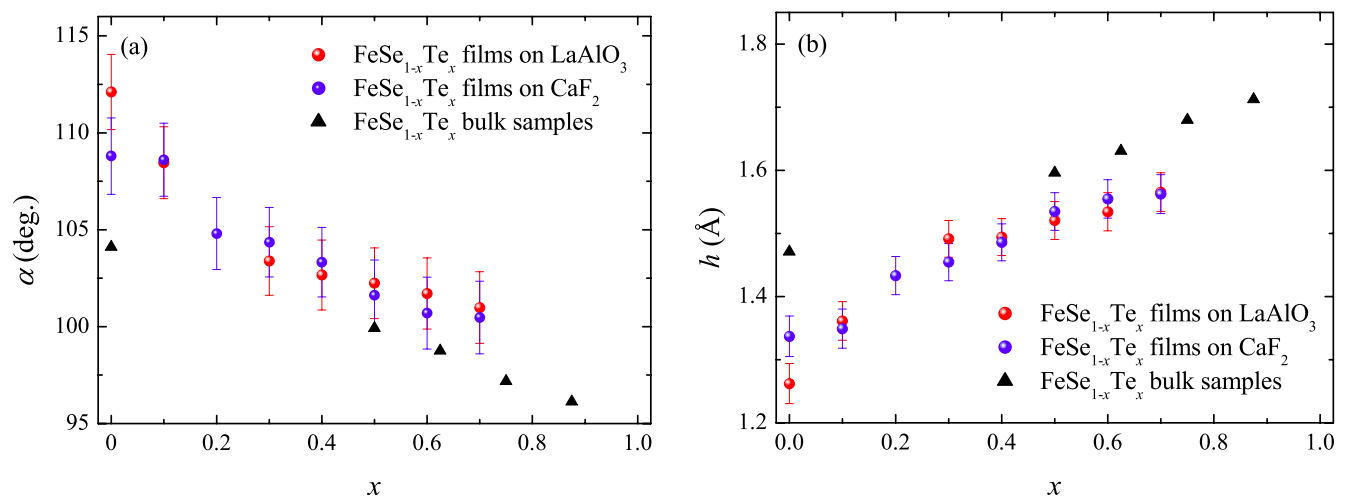

Figure 7. Te content dependence of (a) bond angle, $\alpha$; and (b) Chalcogen height, $h$, for $\mathrm{FeSe}_{1-x} \mathrm{Te}_{x}$ films on $\mathrm{CaF}_{2}$ and LAO substrates. The data for $\mathrm{FeSe}_{1-x} \mathrm{Te}_{x}$ bulk samples [210] are also shown, for comparison. 

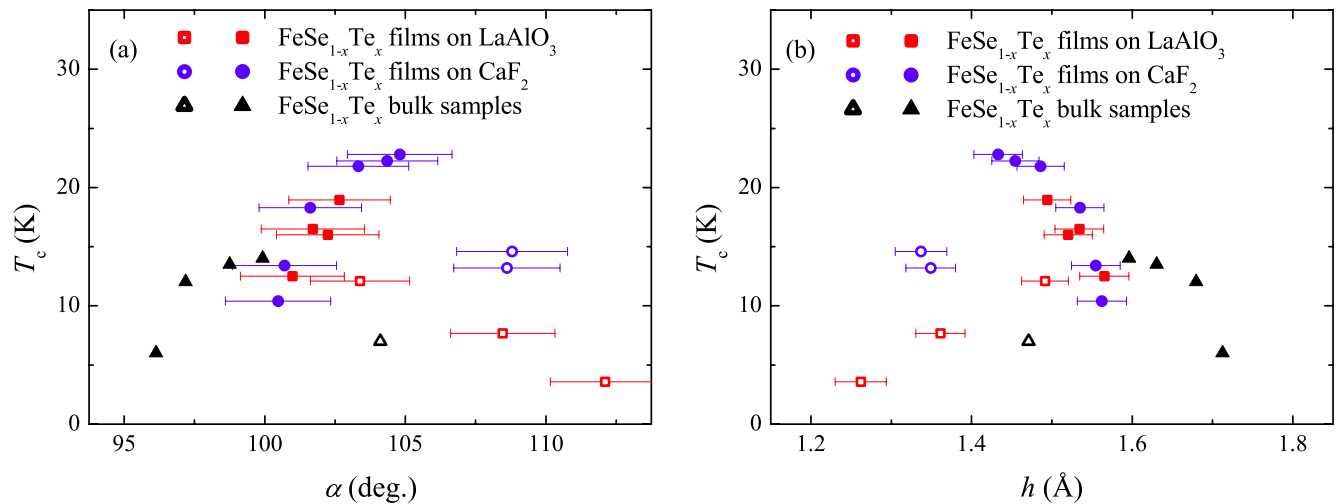

Figure 8. Relation between $T_{\mathrm{c}}$ and (a) bond angle, $\alpha$; and (b) Chalcogen height, $h$, for $\mathrm{FeSe}_{1-x} \mathrm{Te}_{x}$ films on $\mathrm{LaAlO}_{3}$ (red symbols) and $\mathrm{CaF}_{2}$ (blue symbols) substrates. The data for $\mathrm{FeSe}_{1-x} \mathrm{Te}_{x}$ bulk samples [210] are also shown, for comparison. The open (closed) symbols corresponds to the samples with (without) structural transitions.

At $x_{\mathrm{opt}}$, which is closely related to the structural/nematic transition, big changes are observed in some physical properties other than $T_{\mathrm{c}}$ : the upper critical field, $B_{\mathrm{c} 2}$ [209], the superconducting transition width [213], the ratio of $B_{\mathrm{c} 2}$ along the $a b$-plane and the $c$-axis in the orbital limit, $\gamma \equiv B_{\mathrm{c} 2,0 \mathrm{~K}}^{/ / a b} / B_{\mathrm{c} 2,0 \mathrm{~K}}^{/ / c}[213]$, the temperature dependence of Hall coefficient, $R_{\mathrm{H}}(T)$ [209], and the optical conductivity [214]. In Figure $5 \mathrm{c}-\mathrm{f}$, we plot the magnetic field dependence of magneto resistance, $\mathrm{MR}$, at different temperatures, which is the so-called Kohler plot, where the MR can be scaled by $\rho(H, T) / \rho(H=0, T)=F\left(\omega_{c} \tau\right)=$ $F\left(\mu_{0} H / \rho(H=0, T)\right)$ according to the Kohler's rule. Here, $F$ is a function of the cyclotron frequency, $\omega_{c}$, and the relaxation time, $\tau$, which depend on the nature of the materials. At $T>T_{s}$ in Figure $5 c-e$ and all temperatures in Figure $5 f$, the MR is small and can be almost scaled by one curve. On the other hand, the values of MR rapidly increases with decreasing temperature below $T_{\mathrm{S}}$ and are against the Kohler's rule at $T<T_{S}$ in Figure $5 \mathrm{c}-\mathrm{e}$. This indicates that the scattering rate is anisotropic at the Fermi surface below $T_{\mathrm{s}}$. In FeSe bulk single crystals, the similar violence of the Kohler's rule is observed at low temperatures [215].

Finally, we would like to compare our phase diagram of Figure $3 b$ to those obtained in bulk samples of 11 materials. In bulk single crystals, the detailed phase diagram of FeSe under hydrostatic pressures [216-218] and $\mathrm{FeSe}_{1-x} \mathrm{~S}_{x}[219,220]$ have been reported because the high-quality single crystals of $\mathrm{FeSe}_{1-x} \mathrm{~S}_{x}$ and FeSe can be available. The S-substitution for Se introduces chemical pressure in FeSe, and $T_{\mathrm{S}}$ gradually decreases with increasing $S$ content $x$ [219]. The structural/nematic transition is completely suppressed at $x=0.17$, where the nematic fluctuations were found to diverge toward $T \rightarrow 0 \mathrm{~K}$ from the measurements of nematic susceptibility [219]. This result shows that the nematic quantum critical point (QCP) exists around $x=0.17$ [219]. A superconducting transition temperature slightly increases by the $\mathrm{S}$ substitution, and the highest $T_{\mathrm{C}}$ is obtained deep inside the nematic ordered phase [219]. Thus, Hosoi et al. insisted that the critical nematic fluctuations do not have a simple correlation with the $T_{\mathrm{c}}$ enhancement [219]. Contrary to the phase diagram of $\mathrm{FeSe}_{1-x} \mathrm{~S}_{x}$, in $\mathrm{FeSe}_{1-x} \mathrm{Te}_{x}$ thin films (Figure 3), the values of $T_{\mathrm{c}}$ suddenly change around $x_{\mathrm{opt}}$ where the structural/nematic transition disappears. At this moment, it is not clear whether a nematic QCP exists around $x_{\mathrm{opt}}$ or not. However, if it is revealed that the nematic QCP exists around $x_{\text {opt }}$ by evaluating the nematic fluctuations in $\mathrm{FeSe}_{1-x} \mathrm{Te}_{x}$ films as Ba122 [221,222] and $\mathrm{FeSe}_{1-x} \mathrm{~S}_{x}$ [219], it can be concluded that the nematic fluctuation may enhance a superconducting transition temperature as suggested by some theoretical studies [223]. On the other hand, in the phase diagram of FeSe under hydrostatic pressures, $T_{\mathrm{S}}$ also decreases with increasing applied pressure and disappears around $2 \mathrm{GPa}$ [217]. Surprisingly, the magnetic order emerges around $1 \mathrm{GPa}[224,225]$. The magnetic transition temperature $\left(T_{\mathrm{m}}\right)$ increases up to $45 \mathrm{~K}$ with increasing pressure, and then $T_{\mathrm{m}}$ disappears around $6 \mathrm{GPa}$ [217]. Interestingly, in $T_{\mathrm{c}}$ for FeSe under hydrostatic pressures, a characteristic three-step increase is observed [216]; the 2nd 
and 3rd increases in $T_{\mathrm{C}}$ appear around $2 \mathrm{GPa}$ and $6 \mathrm{GPa}$, respectively. The 2nd increase in $T_{\mathrm{c}}$ is closely related with the suppression of $T_{\mathrm{S}}$ [217], which is similar to the rapid increase of $T_{\mathrm{C}}$ at $x_{\mathrm{opt}}$ in $\mathrm{FeSe}_{1-x} \mathrm{Te}_{x}$ thin films (Figure 3), while there are no clear evidences for the existence of a magnetic order in $\mathrm{FeSe}_{1-x} \mathrm{Te}_{x}$ films. For clarifying the differences in these three phase diagrams, we consider that it is of crucial importance to reveal whether $x_{\text {opt }}$ in the phase diagram of $\mathrm{FeSe}_{1-x} \mathrm{Te}_{x}$ (Figure $3 \mathrm{~b}$ ) is a nematic QCP or not, and that the measurement of the nematic and/or superconducting fluctuations in $\mathrm{FeSe}_{1-x} \mathrm{Te}_{x}$ films [226] is a key experiment for realizing it.

\section{Conclusions}

We reviewed the progress of the works on the film fabrications of iron-based superconductors. Initially, there were some difficulties in the film growth. However, its technology has much advanced in the last nine years. In 1111, 122, and 11 systems, the epitaxial films with higher $T_{\mathrm{C}}$ than those of bulk samples have been fabricated. Surprisingly, in these three systems whose in-plane lattice parameters rather differ from each other, the highest $T_{\mathrm{C}}$ was realized in films on the same substrate material, $\mathrm{CaF}_{2}$, which is probably related to the large thermal expansion of $\mathrm{CaF}_{2}$. Additionally, the values of $J_{\mathrm{c}}$ for these films are also large even at high magnetic fields, which the fabrication technique of iron-based superconductor films with high quality is expected to apply the fabrications of the advanced device.

There are many interesting phenomena, for example (1) high $T_{\mathrm{c}}$ in FeSe monolayer film; (2) strain-induced superconductivity (3) novel material obtained only by the film technique, and so on, observed in films of iron-based superconductors. Especially, in the FeSe monolayer film, there is a possibility that the $T_{\mathrm{C}}$ values exceeds boiling point of liquid nitrogen and that the record of $T_{\mathrm{C}}$ in iron-based superconductors is broken. On the other hand, in $\mathrm{FeSe}_{1-x} \mathrm{Te}_{x}$ thin films, it is revealed that the phase separation can be suppressed, which enables us to use the whole phase diagram in this system. The large enhancement of $T_{\mathrm{C}}$ is observed in the conventional phase separation region. More importantly, the suppression of phase diagram opens an avenue for investigating the composition dependences of many physical properties, for example, the optical conductivity, the electronic structure, the nematic and superconducting fluctuations, and so on. Some of these measurements are currently underway, which will be shown in the future papers. Including the works on $\mathrm{FeSe}_{1-x} \mathrm{Te}_{x}$ films, some of researches concerning iron-based superconducting films are still under debate, which are considered to be key issues for revealing the mechanism of the unconventional superconductivity in iron-based superconductors.

Acknowledgments: The authors acknowledge collaborations with Takanori Akiike, Ryo Tanaka, Yuichi Sawada, Daisuke Asami, Masataka Kawai, Daisuke Nakamura, Hideyuki Takahashi, Tatsunori Okada, Ryo Ogawa, Masafumi Hanawa, Seiki Komiya, Ataru Ichinose, Ichiro Tsukada, Tatsuo Hikage, Takahiko Kawaguchi, Hiroshi Ikuta, Takayoshi Katase, Hidenori Hiramatsu, Hideo Hosono, Dario Daghero, Paola Pecchio, Giovanni Ummarino, Renato Gonnelli, Costantino Mauro, Carlo Barone, Sergio Pagano, Masamichi Nakajima, Kazuya Yanase, Setsuko Tajima, Giao N. Phan, Kosuke Nakayama, Katsuaki Sugawara, Takafumi Sato, and Takashi Takahashi. This work was supported by JSPS KAKENHI Grant Numbers 15K17697 and 14J09315.

Author Contributions: Yoshinori Imai, Fuyuki Nabeshima and Atsutaka Maeda conceived and designed the experiments; Yoshinori Imai and Fuyuki Nabeshima performed the experiments and analyzed the data; Yoshinori Imai, Fuyuki Nabeshima and Atsutaka Maeda wrote the paper. All authors have read and approved the final manuscript.

Conflicts of Interest: The authors declare no conflict of interest.

\section{References}

1. Kamihara, Y.; Hiramatsu, H.; Hirano, M.; Kawamura, R.; Yanagi, H.; Kamiya, T.; Hosono, H. Iron-Based Layered Superconductor: LaOFeP. J. Am. Chem. Soc. 2006, 128, 10012.

2. Kamihara, Y.; Watanabe, T.; Hirano, M.; Hosono, H. Iron-Based Layered Superconductor $\mathrm{La}\left(\mathrm{O}_{1-x} \mathrm{~F}_{x}\right) \mathrm{Fe} A s$ $(x=0.05-0.12)$ with $T_{\mathrm{C}}=26$ K. J. Am. Chem. Soc. 2008, 130, 3296. 
3. Ren, Z.A.; Lu, W.; Yang, J.; Yi, W.; Shen, X.L.; Li, Z.C.; Che, G.C.; Dong, X.L.; Sun, L.L.; Zhou, F.; et al. Superconductivity at $55 \mathrm{~K}$ in Iron-Based F-Doped Layered Quaternary Compound $\mathrm{Sm}\left[\mathrm{O}_{1-x} \mathrm{~F}_{x}\right] \mathrm{FeAs}$. Chin. Phys. Lett. 2008, 25, 2215.

4. Hosono, H.; Kuroki, K. Iron-based superconductors: Current status of materials and pairing mechanism. Physica C 2015, 514, 399-422.

5. Singh, D.J.; Du, M.H. Density Functional Study of $\mathrm{LaFeAsO}_{1-x} \mathrm{~F}_{x}$ : A Low Carrier Density Superconductor Near Itinerant Magnetism. Phys. Rev. Lett. 2008, 100, 237003.

6. Subedi, A.; Zhang, L.; Singh, D.J.; Du, M.H. Density functional study of FeS, FeSe, and FeTe: Electronic structure, magnetism, phonons, and superconductivity. Phys. Rev. B 2008, 78, 134514.

7. Ishibashi, S.; Terakura, K.; Hosono, H. A Possible Ground State and Its Electronic Structure of a Mother Material (LaOFeAs) of New Superconductors. J. Phys. Soc. Jpn. 2008, 77, 053709.

8. Mazin, I.I.; Singh, D.J.; Johannes, M.D.; Du, M.H. Unconventional Superconductivity with a Sign Reversal in the Order Parameter of $\mathrm{LaFeAsO}_{1-x} \mathrm{~F}_{x}$. Phys. Rev. Lett. 2008, 101, 057003.

9. Kuroki, K.; Onari, S.; Arita, R.; Usui, H.; Tanaka, Y.; Kontani, H.; Aoki, H. Unconventional Pairing Originating from the Disconnected Fermi Surfaces of Superconducting $\mathrm{LaFeAsO}_{1-x} \mathrm{~F}_{x}$. Phys. Rev. Lett. 2008, 101, 087004.

10. Yanagi, Y.; Yamakawa, Y.; Ono, Y. Two types of s-wave pairing due to magnetic and orbital fluctuations in the two-dimensional 16-band $d-p$ model for iron-based superconductors. Phys. Rev. B 2010, 81, 054518.

11. Kontani, H.; Onari, S. Orbital-Fluctuation-Mediated Superconductivity in Iron Pnictides: Analysis of the Five-Orbital Hubbard-Holstein Model. Phys. Rev. Lett. 2010, 104, 157001.

12. Yanagi, Y.; Yamakawa, Y.; Adachi, N.; Ono, Y. Orbital Order, Structural Transition, and Superconductivity in Iron Pnictides. J. Phys. Soc. Jpn. 2010, 79, 123707.

13. Liu, D.; Zhang, W.; Mou, D.; He, J.; Ou, Y.B.; Wang, Q.Y.; Li, Z.; Wang, L.; Zhao, L.; He, S.; et al. Electronic origin of high-temperature superconductivity in single-layer FeSe superconductor. Nat. Commun. 2012, $3,931$.

14. Zhang, Y.; Yang, L.X.; Xu, M.; Ye, Z.R.; Chen, F.; He, C.; Xu, H.C.; Jiang, J.; Xie, B.P.; Ying, J.J.; et al. Nodeless superconducting gap in $A_{x} \mathrm{Fe}_{2} \mathrm{Se}_{2}(A=\mathrm{K}, \mathrm{Cs})$ revealed by angle-resolved photoemission spectroscopy. Nat. Mater. 2011, 10, 273.

15. Qian, T.; Wang, X.P.; Jin, W.C.; Zhang, P.; Richard, P.; Xu, G.; Dai, X.; Fang, Z.; Guo, J.G.; Chen, X.L.; et al. Absence of a Holelike Fermi Surface for the Iron-Based $\mathrm{K}_{0.8} \mathrm{Fe}_{1.7} \mathrm{Se}_{2}$ Superconductor Revealed by Angle-Resolved Photoemission Spectroscopy. Phys. Rev. Lett. 2011, 106, 187001.

16. Kirtley, J.R.; Tsuei, C.C.; Sun, J.Z.; Chi, C.C.; Yu-Jahnes, L.S.; Gupta, A.; Rupp, M.; Ketchen, M.B. Symmetry of the order parameter in the high- $T_{\mathrm{c}}$ superconductor $\mathrm{YBa}_{2} \mathrm{Cu}_{3} \mathrm{O}_{7-\delta}$. Nature 1995, 373, 225.

17. Tsuei, C.C.; Kirtley, J.R. Pairing symmetry in cuprate superconductors. Rev. Mod. Phys. 2000, 72, $969-1016$.

18. Hsu, F.C.; Luo, J.Y.; Yeh, K.W.; Chen, T.K.; Huang, T.W.; Wu, P.M.; Lee, Y.C.; Huang, Y.L.; Chu, Y.Y.; Yan, D.C.; et al. Superconductivity in the PbO-type structure $\alpha$-FeSe. Proc. Natl. Acad. Sci. USA 2008, $105,14262$.

19. Putti, M.; Pallecchi, I.; Bellingeri, E.; Cimberle, M.R.; Tropeano, M.; Ferdeghini, C.; Palenzona, A.; Tarantini, C.; Yamamoto, A.; Jiang, J.; et al. New Fe-based superconductors: Properties relevant for applications. Supercond. Sci. Technol. 2010, 23, 034003.

20. Li, Q.; Si, W.; Dimitrov, I.K. Films of iron chalcogenide superconductors. Rep. Prog. Phys. 2011, 74, 124510.

21. Hiramatsu, H.; Katase, T.; Kamiya, T.; Hosono, H. Thin Film Growth and Device Fabrication of Iron-Based Superconductors. J. Phys. Soc. Jpn. 2012, 81, 011011.

22. Tanabe, K.; Hosono, H. Frontiers of Research on Iron-Based Superconductors toward Their Application. Jpn. J. Appl. Phys. 2012, 51, 010005.

23. Mele, P. Superconducting properties of iron chalcogenide thin films. Sci. Technol. Adv. Mater. 2012, 13, 054301.

24. Haindl, S.; Kidszun, M.; Oswald, S.; Hess, C.; Büchner, B.; Kölling, S.; Wilde, L.; Thersleff, T.; Yurchenko, V.V.; Jourdan, M.; et al. Thin film growth of Fe-based superconductors: from fundamental properties to functional devices. A comparative review. Rep. Prog. Phys. 2014, 77, 046502.

25. Haindl, S.; Molatta, S.; Hiramatsu, H.; Hosono, H. Recent progress in pulsed laser deposition of iron based superconductors. J. Phys. D Appl. Phys. 2016, 49, 345301.

26. Haindl, S.; Kidszun, M.; Kampert, E. Iron pnictide thin films: Synthesis and physics. Phys. Status Solidi (b) 2016, 254, 1600341. 
27. Fang, M.H.; Pham, H.M.; Qian, B.; Liu, T.J.; Vehstedt, E.K.; Liu, Y.; Spinu, L.; Mao, Z.Q. Superconductivity close to magnetic instability in $\mathrm{Fe}\left(\mathrm{Se}_{1-x} \mathrm{Te}_{x}\right)_{0.82}$. Phys. Rev. B 2008, 78, 224503.

28. Hiramatsu, H.; Katase, T.; Kamiya, T.; Hirano, M.; Hosono, H. Heteroepitaxial growth and optoelectronic properties of layered iron oxyarsenide, LaFeAsO. Appl. Phys. Lett. 2008, 93, 162504.

29. De la Cruz, C.; Huang, Q.; Lynn, J.W.; Li, J.; Ratcliff, W., II; Zarestky, J.L.; Mook, H.A.; Chen, G.F.; Luo, J.L.; Wang, N.L.; et al. Magnetic order close to superconductivity in the iron-based layered $\mathrm{LaO}_{1-x} \mathrm{~F}_{x} \mathrm{FeAs}$ systems. Nature 2008, 453, 899.

30. Hiramatsu, H.; Katase, T.; Kamiya, T.; Hirano, M.; Hosono, H. Superconductivity in Epitaxial Thin Films of Co-Doped $\mathrm{SrFe}_{2} \mathrm{As}_{2}$ with Bilayered FeAs Structures and their Magnetic Anisotropy. Appl. Phys. Expr. 2008, 1, 101702.

31. Haindl, S.; Hanzawa, K.; Sato, H.; Hiramatsu, H.; Hosono, H. In-situ growth of superconducting $\mathrm{SmO}_{1-x} \mathrm{~F}_{x} \mathrm{FeAs}$ thin films by pulsed laser deposition. Sci. Rep. 2016, 6, 35797.

32. Backen, E.; Haindl, S.; Niemeier, T.; Hühne, R.; Freudenberg, T.; Werner, J.; Behr, G.; Schultz, L.; Holzapfel, B. Growth and anisotropy of $\mathrm{La}(\mathrm{O}, \mathrm{F}) \mathrm{FeAs}$ thin films deposited by pulsed laser deposition. Supercond. Sci. Technol. 2008, 21, 122001.

33. Haindl, S.; Kidszun, M.; Kauffmann, A.; Nenkov, K.; Kozlova, N.; Freudenberger, J.; Thersleff, T.; Hänisch, J.; Werner, J.; Reich, E.; et al. High Upper Critical Fields and Evidence of Weak-Link Behavior in Superconducting $\mathrm{LaFeAsO}_{1-x} \mathrm{~F}_{x}$ Thin Films. Phys. Rev. Lett. 2010, 104, 077001.

34. Kidszun, M.; Haindl, S.; Reich, E.; Hänisch, J.; Iida, K.; Schultz, L.; Holzapfel, B. Epitaxial LaFeAsO $\mathrm{O}_{1-x} \mathrm{~F}_{x}$ thin films grown by pulsed laser deposition. Supercond. Sci. Technol. 2010, 23, 022002.

35. Kawaguchi, T.; Uemura, H.; Ohno, T.; Watanabe, R.; Tabuchi, M.; Ujihara, T.; Takenaka, K.; Takeda, Y.; Ikuta, H. Epitaxial Growth of NdFeAsO Thin Films by Molecular Beam Epitaxy. Appl. Phys. Expr. 2009, 2, 093002.

36. Kawaguchi, T.; Uemura, H.; Ohno, T.; Tabuchi, M.; Ujihara, T.; Takenaka, K.; Takeda, Y.; Ikuta, H. In situ growth of superconducting $\operatorname{NdFeAs}(\mathrm{O}, \mathrm{F})$ thin films by molecular beam epitaxy. Appl. Phys. Lett. 2010, 97, 042509 .

37. Kawaguchi, T.; Uemura, H.; Ohno, T.; Tabuchi, M.; Ujihara, T.; Takeda, Y.; Ikuta, H. Molecular Beam Epitaxy Growth of Superconducting $\operatorname{NdFeAs}(\mathrm{O}, \mathrm{F})$ Thin Films Using a F-Getter and a Novel F-Doping Method. Appl. Phys. Expr. 2011, 4, 083102.

38. Ueda, S.; Takeda, S.; Takano, S.; Yamamoto, A.; Naito, M. High- $T_{\mathrm{C}}$ and high- $J_{\mathrm{C}} \mathrm{SmFeAs}(\mathrm{O}, \mathrm{F})$ films on fluoride substrates grown by molecular beam epitaxy. Appl. Phys. Lett. 2011, 99, 232505.

39. Kamihara, Y.; Nomura, T.; Hirano, M.; Kim, J.E.; Kato, K.; Takata, M.; Kobayashi, Y.; Kitao, S.; Higashitaniguchi, S.; Yoda, Y.; et al. Electronic and magnetic phase diagram of superconductors, $\mathrm{SmFeAsO}_{1-x} \mathrm{~F}_{x}$. New J. Phys. 2010, 12, 033005.

40. Tsukada, I.; Hanawa, M.; Akiike, T.; Nabeshima, F.; Imai, Y.; Ichinose, A.; Komiya, S.; Hikage, T.; Kawaguchi, T.; Ikuta, H.; et al. Epitaxial Growth of $\mathrm{FeSe}_{0.5} \mathrm{Te}_{0.5}$ Thin Films on $\mathrm{CaF}_{2}$ Substrates with High Critical Current Density. Appl. Phys. Expr. 2011, 4, 053101.

41. Ueda, S.; Takeda, S.; Takano, S.; Naito, M. As-Grown Superconducting $\operatorname{SmFeAs}(\mathrm{O}, \mathrm{F})$ Thin Films by Molecular Beam Epitaxy. Appl. Phys. Expr. 2012, 5, 053101.

42. Sugawara, H.; Tsuneki, T.; Watanabe, D.; Yamamoto, A.; Sakoda, M.; Naito, M. One-step growth of $\mathrm{SmFeAs}(\mathrm{O}, \mathrm{F})$ films by molecular beam epitaxy using $\mathrm{FeF}_{2}$ as a fluorine source. Supercond. Sci. Technol. 2015, 28,015005 .

43. Chihara, M.; Sumiya, N.; Arai, K.; Ichinose, A.; Tsukada, I.; Hatano, T.; Iida, K.; Ikuta, H. Direct growth of superconducting $\mathrm{NdFeAs}(\mathrm{O}, \mathrm{F})$ thin films by MBE. Physica C 2015, 518, 69.

44. Iida, K.; Kurth, F.; Chihara, M.; Sumiya, N.; Grinenko, V.; Ichinose, A.; Tsukada, I.; Hänisch, J.; Matias, V.; Hatano, T.; et al. Highly textured oxypnictide superconducting thin films on metal substrates. Appl. Phys. Lett. 2014, 105, 172602.

45. Ichinose, A.; Nabeshima, F.; Tsukada, I.; Hanawa, M.; Komiya, S.; Akiike, T.; Imai, Y.; Maeda, A. Microscopic analysis of the chemical reaction between $\mathrm{Fe}(\mathrm{Te}, \mathrm{Se})$ thin films and underlying $\mathrm{CaF}_{2}$. Supercond. Sci. Technol. 2013, 26, 075002.

46. Ichinose, A.; Tsukada, I.; Nabeshima, F.; Imai, Y.; Maeda, A.; Kurth, F.; Holzapfel, B.; Iida, K.; Ueda, S.; Naito, M. Induced lattice strain in epitaxial Fe-based superconducting films on $\mathrm{CaF}_{2}$ substrates: 
A comparative study of the microstructures of $\mathrm{SmFeAs}(\mathrm{O}, \mathrm{F}), \mathrm{Ba}(\mathrm{Fe}, \mathrm{Co})_{2} \mathrm{As}_{2}$, and $\mathrm{FeTe}_{0.5} \mathrm{Se}_{0.5}$. Appl. Phys. Lett. 2014, 104, 122603.

47. Choi, E.M.; Jung, S.G.; Lee, N.H.; Kwon, Y.S.; Kang, W.N.; Kim, D.H.; Jung, M.H.; Lee, S.I.; Sun, L. In situ fabrication of cobalt-doped $\mathrm{SrFe}_{2} \mathrm{As}_{2}$ thin films by using pulsed laser deposition with excimer laser. Appl. Phys. Lett. 2009, 95, 062507.

48. Hiramatsu, H.; Katase, T.; Kamiya, T.; Hirano, M.; Hosono, H. Water-induced superconductivity in $\mathrm{SrFe}_{2} \mathrm{As}_{2}$. Phys. Rev. B 2009, 80, 052501.

49. Katase, T.; Hiramatsu, H.; Yanagi, H.; Kamiya, T.; Hirano, M.; Hosono, H. Atomically-flat, chemically-stable, superconducting epitaxial thin film of iron-based superconductor, cobalt-doped. Solid State Commun. 2009, $149,2121-2124$.

50. Lee, S.; Jiang, J.; Weiss, J.D.; Folkman, C.M.; Bark, C.W.; Tarantini, C.; Xu, A.; Abraimov, D.; Polyanskii, A.; Nelson, C.T.; et al. Weak-link behavior of grain boundaries in superconducting $\mathrm{Ba}\left(\mathrm{Fe}_{1-x} \mathrm{Co}_{x}\right)_{2} \mathrm{As}_{2}$ bicrystals. Appl. Phys. Lett. 2009, 95, 212505.

51. Iida, K.; Hänisch, J.; Hühne, R.; Kurth, F.; Kidszun, M.; Haindl, S.; Werner, J.; Schultz, L.; Holzapfel, B. Strong $T_{\mathrm{C}}$ dependence for strained epitaxial $\mathrm{Ba}\left(\mathrm{Fe}_{1-x} \mathrm{Co}_{x}\right)_{2} \mathrm{As}_{2}$ thin films. Appl. Phys. Lett. 2009, 95, 192501.

52. Imai, Y.; Akiike, T.; Hanawa, M.; Tsukada, I.; Ichinose, A.; Maeda, A.; Hikage, T.; Kawaguchi, T.; Ikuta, H. Systematic Comparison of Eight Substrates in the Growth of FeSe $\mathrm{F}_{0.5} \mathrm{Te}_{0.5}$ Superconducting Thin Films. Appl. Phys. Express 2010, 3, 043102.

53. Lee, S.; Jiang, J.; Zhang, Y.; Bark, C.W.; Weiss, J.D.; Tarantini, C.; Nelson, C.T.; Jang, H.W.; Folkman, C.M.; Baek, S.H.; et al. Template engineering of Co-doped $\mathrm{BaFe}_{2} \mathrm{As}_{2}$ single-crystal thin films. Nat. Mater. 2010, $9,397$.

54. Iida, K.; Haindl, S.; Thersleff, T.; Hänisch, J.; Kurth, F.; Kidszun, M.; Hühne, R.; Mönch, I.; Schultz, L.; Holzapfel, B.; et al. Influence of Fe buffer thickness on the crystalline quality and the transport properties of $\mathrm{Fe} / \mathrm{Ba}\left(\mathrm{Fe}_{1-x} \mathrm{Co}_{x}\right)_{2} \mathrm{As}_{2}$ bilayers. Appl. Phys. Lett. 2010, 97, 172507.

55. Thersleff, T.; Iida, K.; Haindl, S.; Kidszun, M.; Pohl, D.; Hartmann, A.; Kurth, F.; Hänisch, J.; Hühne, R.; Rellinghaus, B.; et al. Coherent interfacial bonding on the FeAs tetrahedron in $\mathrm{Fe} / \mathrm{Ba}\left(\mathrm{Fe}_{1-x} \mathrm{Co}_{x}\right)_{2} \mathrm{As}_{2}$ bilayers. Appl. Phys. Lett. 2010, 97, 022506.

56. Iida, K.; Hänisch, J.; Trommler, S.; Haindl, S.; Kurth, F.; Hühne, R.; Schultz, L.; Holzapfel, B. Thickness dependence of structural and transport properties of Co-doped $\mathrm{BaFe}_{2} \mathrm{As}_{2}$ on Fe buffered $\mathrm{MgO}$ substrates. Supercond. Sci. Technol. 2011, 24, 125009.

57. Iida, K.; Hänisch, J.; Thersleff, T.; Kurth, F.; Kidszun, M.; Haindl, S.; Hühne, R.; Schultz, L.; Holzapfel, B. Scaling behavior of the critical current in clean epitaxial $\mathrm{Ba}\left(\mathrm{Fe}_{1-x} \mathrm{Co}_{x}\right)_{2} \mathrm{As}_{2}$ thin films. Phys. Rev. B 2010, 81, 100507.

58. Katase, T.; Ishimaru, Y.; Tsukamoto, A.; Hiramatsu, H.; Kamiya, T.; Tanabe, K.; Hosono, H. Josephson junction in cobalt-doped $\mathrm{BaFe}_{2} \mathrm{As}_{2}$ epitaxial thin films on $(\mathrm{La}, \mathrm{Sr})(\mathrm{Al}, \mathrm{Ta}) \mathrm{O}_{3}$ bicrystal substrates. Appl. Phys. Lett. 2010, 96, 142507.

59. Katase, T.; Ishimaru, Y.; Tsukamoto, A.; Hiramatsu, H.; Kamiya, T.; Tanabe, K.; Hosono, H. DC superconducting quantum interference devices fabricated using bicrystal grain boundary junctions in Co-doped $\mathrm{BaFe}_{2} \mathrm{As}_{2}$ epitaxial films. Supercond. Sci. Technol. 2010, 23, 082001.

60. Schmidt, S.; Döring, S.; Schmidl, F.; Grosse, V.; Seidel, P.; Iida, K.; Kurth, F.; Haindl, S.; Mönch, I.; Holzapfel, B. $\mathrm{BaFe}_{1.8} \mathrm{Co}_{0.2} \mathrm{As}_{2}$ thin film hybrid Josephson junctions. Appl. Phys. Lett. 2010, 97, 172504.

61. Sefat, A.S.; Jin, R.; McGuire, M.A.; Sales, B.C.; Singh, D.J.; Mandrus, D. Superconductivity at 22 K in Co-Doped $\mathrm{BaFe}_{2} \mathrm{As}_{2}$ Crystals. Phys. Rev. Lett. 2008, 101, 117004.

62. Ishida, S.; Nakajima, M.; Liang, T.; Kihou, K.; Lee, C.H.; Iyo, A.; Eisaki, H.; Kakeshita, T.; Tomioka, Y.; Ito, T.; et al. Effect of Doping on the Magnetostructural Ordered Phase of Iron Arsenides: A Comparative Study of the Resistivity Anisotropy in Doped $\mathrm{BaFe}_{2} \mathrm{As}_{2}$ with Doping into Three Different Sites. J. Am. Chem. Soc. 2013, 135, 3158-3163.

63. Kurth, F.; Reich, E.; Hänisch, J.; Ichinose, A.; Tsukada, I.; Hühne, R.; Trommler, S.; Engelmann, J.; Schultz, L.; Holzapfel, B.; et al. Versatile fluoride substrates for Fe-based superconducting thin films. Appl. Phys. Lett. 2013, 102, 142601.

64. Rotter, M.; Tegel, M.; Johrendt, D. Superconductivity at $38 \mathrm{~K}$ in the Iron Arsenide $\left(\mathrm{Ba}_{1-x} \mathrm{~K}_{x}\right) \mathrm{Fe}_{2} \mathrm{As}_{2}$. Phys. Rev. Lett. 2008, 101, 107006. 
65. Takeda, S.; Ueda, S.; Yamagishi, T.; Agatsuma, S.; Takano, S.; Mitsuda, A.; Naito, M. Molecular Beam Epitaxy Growth of Superconducting $\mathrm{Sr}_{1-x} \mathrm{~K}_{x} \mathrm{Fe}_{2} \mathrm{As}_{2}$ and $\mathrm{Ba}_{1-x} \mathrm{~K}_{x} \mathrm{Fe}_{2} \mathrm{As}_{2}$. Appl. Phys. Express 2010, 3, 093101.

66. Agatsuma, S.; Yamagishi, T.; Takeda, S.; Naito, M. MBE growth of FeSe and $\mathrm{Sr}_{1-x} \mathrm{~K}_{x} \mathrm{Fe}_{2} \mathrm{As}_{2}$. Physica C 2010, $470,1468$.

67. Yamagishi, T.; Ueda, S.; Takeda, S.; Takano, S.; Mitsuda, A.; Naito, M. A study of the doping dependence of $T_{\mathrm{C}}$ in $\mathrm{Ba}_{1-x} \mathrm{~K}_{x} \mathrm{Fe}_{2} \mathrm{As}_{2}$ and $\mathrm{Sr}_{1-x} \mathrm{~K}_{x} \mathrm{Fe}_{2} \mathrm{As}_{2}$ films grown by molecular beam epitaxy. Physica $C$ 2011, $471,1177-1180$.

68. Lee, N.H.; Jung, S.G.; Kim, D.H.; Kang, W.N. Potassium-doped BaFe $\mathrm{As}_{2}$ superconducting thin films with a transition temperature of 40 K. Appl. Phys. Lett. 2010, 96, 202505.

69. Yuan, H.Q.; Singleton, J.; Balakirev, F.F.; Baily, S.A.; Chen, G.F.; Luo, J.L.; Wang, N.L. Nearly isotropic superconductivity in $(\mathrm{Ba}, \mathrm{K}) \mathrm{Fe}_{2} \mathrm{As}_{2}$. Nature 2009, 457, 565.

70. Yamamoto, A.; Jaroszynski, J.; Tarantini, C.; Balicas, L.; Jiang, J.; Gurevich, A.; Larbalestier, D.C.; Jin, R.; Sefat, A.S.; McGuire, M.A.; et al. Small anisotropy, weak thermal fluctuations, and high field superconductivity in Co-doped iron pnictide $\mathrm{Ba}\left(\mathrm{Fe}_{1-x} \mathrm{Co}_{x}\right)_{2} \mathrm{As}_{2}$. Appl. Phys. Lett. 2009, 94, 062511.

71. Vinod, K.; Satya, A.T.; Sharma, S.; Sundar, C.S.; Bharathi, A. Upper critical field anisotropy in $\mathrm{BaFe}_{2-x} \mathrm{Co}_{x} \mathrm{As}_{2}$ single crystals synthesized without flux. Phys. Rev. B 2011, 84, 012502.

72. Jiang, S.; Xing, H.; Xuan, G.; Wang, C.; Ren, Z.; Feng, C.; Dai, J.; Xu, Z.; Cao, G. Superconductivity up to $30 \mathrm{~K}$ in the vicinity of the quantum critical point in $\mathrm{BaFe}_{2}\left(\mathrm{As}_{1-x} \mathrm{P}_{x}\right)_{2}$. J. Phys. Condens. Matter. 2009, 21, 382203.

73. Adachi, S.; Shimode, T.; Miura, M.; Chikumoto, N.; Takemori, A.; Nakao, K.; Oshikubo, Y.; Tanabe, K. Pulsed laser deposition of $\mathrm{BaFe}_{2}(\mathrm{As}, \mathrm{P})_{2}$ superconducting thin films with high critical current density. Supercond. Sci. Technol. 2012, 25, 105015.

74. Miura, M.; Maiorov, B.; Kato, T.; Shimode, T.; Wada, K.; Adachi, S.; Tanabe, K. Strongly enhanced flux pinning in one-step deposition of $\mathrm{BaFe}_{2}\left(\mathrm{As}_{0.66} \mathrm{P}_{0.33}\right)_{2}$ superconductor films with uniformly dispersed $\mathrm{BaZrO}_{3}$ nanoparticles. Nat. Commun. 2013, 4, 2499.

75. Sakagami, A.; Kawaguchi, T.; Tabuchi, M.; Ujihara, T.; Takeda, Y.; Ikuta, H. Critical current density and grain boundary property of $\mathrm{BaFe}_{2}(\mathrm{As}, \mathrm{P})_{2}$ thin films. Physica C 2013, 494, 181-184.

76. Sato, H.; Hiramatsu, H.; Kamiya, T.; Hosono, H. High critical-current density with less anisotropy in $\mathrm{BaFe}_{2}(\mathrm{As}, \mathrm{P})_{2}$ epitaxial thin films: Effect of intentionally grown $c$-axis vortex-pinning centers. Appl. Phys. Lett. 2014, 104, 182603.

77. Hilgenkamp, H.; Mannhart, J. Grain boundaries in high- $T_{c}$ superconductors. Rev. Mod. Phys. 2002, 74, 485-549.

78. Katase, T.; Ishimaru, Y.; Tsukamoto, A.; Hiramatsu, H.; Kamiya, T.; Tanabe, K.; Hosono, H. Advantageous grain boundaries in iron pnictide superconductors. Nat. Commun. 2011, 2, 409.

79. Katase, T.; Iimura, S.; Hiramatsu, H.; Kamiya, T.; Hosono, H. Identical effects of indirect and direct electron doping of superconducting $\mathrm{BaFe}_{2} \mathrm{As}_{2}$ thin films. Phys. Rev. B 2012, 85, 140516.

80. Malaeb, W.; Shimojima, T.; Ishida, Y.; Okazaki, K.; Ota, Y.; Ohgushi, K.; Kihou, K.; Saito, T.; Lee, C.H.; Ishida, S.; et al. Abrupt change in the energy gap of superconducting $\mathrm{Ba}_{1-x} \mathrm{~K}_{x} \mathrm{Fe}_{2} \mathrm{As}_{2}$ single crystals with hole doping. Phys. Rev. B 2012, 86, 165117.

81. Liu, C.; Kondo, T.; Fernandes, R.M.; Palczewski, A.D.; Mun, E.D.; Ni, N.; Thaler, A.N.; Bostwick, A.; Rotenberg, E.; Schmalian, J.; et al. Evidence for a Lifshitz transition in electron-doped iron arsenic superconductors at the onset of superconductivity. Nat. Phys. 2010, 6, 419-423.

82. Engelmann, J.; Grinenko, V.; Chekhonin, P.; Skrotzki, W.; Efremov, D.; Oswald, S.; Iida, K.; Hühne, R.; Hänisch, J.; Hoffmann, M.; et al. Strain induced superconductivity in the parent compound $\mathrm{BaFe}_{2} \mathrm{As}_{2}$. Nat. Commun. 2013, 4, 2877.

83. Han, Y.; Li, W.Y.; Cao, L.X.; Zhang, S.; Xu, B.; Zhao, B.R. Preparation and superconductivity of iron selenide thin films. J. Phys. Condens. Matter 2009, 21, 235702.

84. Wu, M.K.; Hsu, F.C.; Yeh, K.W.; Huang, T.W.; Luo, J.Y.; Wang, M.J.; Chang, H.H.; Chen, T.K.; Rao, S.M.; Mok, B.H.; et al. The development of the superconducting PbO-type $\beta$-FeSe and related compounds. Physica C 2009, 469, 340-349.

85. Nie, Y.F.; Brahimi, E.; Budnick, J.I.; Hines, W.A.; Jain, M.; Wells, B.O. Suppression of superconductivity in FeSe films under tensile strain. Appl. Phys. Lett. 2009, 94, 242505. 
86. Kumary, T.G.; Baisnab, D.K.; Janaki, J.; Mani, A.; Satya, A.T.; Sarguna, R.M.; Ajikumar, P.K.; Tyagi, A.K.; Bharathi, A. Superconducting $\mathrm{Fe}_{1+\delta} \mathrm{Se}_{1-x} \mathrm{Te}_{x}$ thin films: growth, characterization and properties. Supercond. Sci. Technol. 2009, 22, 095018.

87. Bellingeri, E.; Buzio, R.; Gerbi, A.; Marrè, D.; Congiu, S.; Cimberle, M.R.; Tropeano, M.; Siri, A.S.; Palenzona, A.; Ferdeghini, C. High quality epitaxial $\mathrm{FeSe}_{0.5} \mathrm{Te}_{0.5}$ thin films grown on $\mathrm{SrTiO}_{3}$ substrates by pulsed laser deposition. Supercond. Sci. Technol. 2009, 22, 105007.

88. Si, W.; Zhou, J.; Jie, Q.; Dimitrov, I.; Solovyov, V.; Johnson, P.D.; Jaroszynski, J.; Matias, V.; Sheehan, C.; Li, Q. Iron-chalcogenide $\mathrm{FeSe}_{0.5} \mathrm{Te}_{0.5}$ coated superconducting tapes for high field applications. Appl. Phys. Lett. 2011, 98, 262509.

89. Mele, P.; Matsumoto, K.; Haruyama, Y.; Mukaida, M.; Yoshida, Y.; Kiss, T. Fabrication of Fe-Te-S Superconducting Epitaxial Thin Films by Pulsed Laser Deposition. Appl. Phys. Expr. 2009, 2, 073002.

90. Imai, Y.; Tanaka, R.; Akiike, T.; Hanawa, M.; Tsukada, I.; Maeda, A. Superconductivity of FeSe $\mathrm{e}_{0.5} \mathrm{Te}_{0.5} \mathrm{Thin}$ Films Grown by Pulsed Laser Deposition. Jpn. J. Appl. Phys. 2010, 49, 023101.

91. Huang, S.X.; Chien, C.L.; Thampy, V.; Broholm, C. Control of Tetrahedral Coordination and Superconductivity in $\mathrm{FeSe}_{0.5} \mathrm{Te}_{0.5}$ Thin Films. Phys. Rev. Lett. 2010, 104, 217002.

92. Bellingeri, E.; Pallecchi, I.; Buzio, R.; Gerbi, A.; Marré, D.; Cimberle, M.R.; Tropeano, M.; Putti, M.; Palenzona, A.; Ferdeghini, C. $T_{\mathrm{C}}=21 \mathrm{~K}$ in epitaxial $\mathrm{FeSe}_{0.5} \mathrm{Te}_{0.5}$ thin films with biaxial compressive strain. Appl. Phys. Lett. 2010, 96, 102512.

93. Nabeshima, F.; Imai, Y.; Hanawa, M.; Tsukada, I.; Maeda, A. Enhancement of the superconducting transition temperature in FeSe epitaxial thin films by anisotropic compression. Appl. Phys. Lett. 2013, 103, 172602.

94. Maeda, A.; Nabeshima, F.; Takahashi, H.; Okada, T.; Imai, Y.; Tsukada, I.; Hanawa, M.; Komiya, S.; Ichinose, A. Synthesis, characterization, Hall effect and $\mathrm{THz}$ conductivity of epitaxial thin films of Fe chalcogenide superconductors. Appl. Sur. Sci. 2014, 312, 43.

95. Lee, C.H.; Iyo, A.; Eisaki, H.; Kito, H.; Teresa Fernandez-Diaz, M.; Ito, T.; Kihou, K.; Matsuhata, H.; Braden, M.; Yamada, K. Effect of Structural Parameters on Superconductivity in Fluorine-Free $L_{n F e A s} \mathrm{O}_{1-y}$ (Ln = La, Nd). J. Phys. Soc. Jpn. 2008, 77, 083704.

96. Lee, C.H.; Kihou, K.; Iyo, A.; Kito, H.; Shirage, P.; Eisaki, H. Relationship between crystal structure and superconductivity in iron-based superconductors. Solid State Commun. 2012, 152, 644.

97. Tsukada, A.; Luna, K.E.; Hammond, R.H.; Beasley, M.R.; Zhao, J.F.; Risbud, S.H. Pulsed laser deposition conditions and superconductivity of FeSe thin films. Appl. Phys. A 2011, 104, 311-318.

98. Wang, Q.Y.; Li, Z.; Zhang, W.H.; Zhang, Z.C.; Zhang, J.S.; Li, W.; Ding, H.; Ou, Y.B.; Deng, P.; Chang, K.; et al. Interface-Induced High-Temperature Superconductivity in Single Unit-Cell FeSe Films on $\mathrm{SrTiO}_{3}$. Chin. Phys. Lett. 2012, 29, 37402.

99. Bellingeri, E.; Kawale, S.; Braccini, V.; Buzio, R.; Gerbi, A.; Martinelli, A.; Putti, M.; Pallecchi, I.; Balestrino, G.; Tebano, A.; et al. Tuning of the superconducting properties of $\mathrm{FeSe}_{0.5} \mathrm{Te}_{0.5}$ thin films through the substrate effect. Supercond. Sci. Technol. 2012, 25, 084022.

100. Hanawa, M.; Ichinose, A.; Komiya, S.; Tsukada, I.; Imai, Y.; Maeda, A. Empirical Selection Rule of Substrate Materials for Iron Chalcogenide Superconducting Thin Films. Jpn. J. Appl. Phys. 2012, 51, 010104.

101. Tsukada, I.; Ichinose, A.; Nabeshima, F.; Imai, Y.; Maeda, A. Origin of lattice compression of FeSe $e_{-x} \mathrm{Te}_{x}$ thin films on $\mathrm{CaF}_{2}$ substrates. AIP Adv. 2016, 6, 095314.

102. Iida, K.; Hänisch, J.; Schulze, M.; Aswartham, S.; Wurmehl, S.; Büchner, B.; Schultz, L.; Holzapfel, B. Generic Fe buffer layers for Fe-based superconductors: Epitaxial $\mathrm{FeSe}_{1-x} \mathrm{Te}_{x}$ thin films. Appl. Phys. Lett. 2011, 99, 202503.

103. Si, W.; Han, S.J.; Shi, X.; Ehrlich, S.N.; Jaroszynski, J.; Goyal, A.; Li, Q. High current superconductivity in $\mathrm{FeSe}_{0.5} \mathrm{Te}_{0.5}$-coated conductors at 30 tesla. Nat. Commun. 2013, 4, 1347.

104. Molatta, S.; Haindl, S.; Trommler, S.; Schulze, M.; Wurmehl, S.; Hühne, R. Interface control by homoepitaxial growth in pulsed laser deposited iron chalcogenide thin films. Sci. Rep. 2015, 5, 16334.

105. Tkachenko, O.; Morawski, A.; Zaleski, A.J.; Przyslupski, P.; Dietl, T.; Diduszko, R.; Presz, A.; Werner-Malento, K. Synthesis, Crystal Growth and Epitaxial Layers Growth of c2 a0FeSe ${ }_{0.88}$ Superconductor and Other Poison Materials byc2a0Use c2a0ofc2a0High Gas Pressure Trap System. J. Supercond. Nov. Magn. 2009, 22, 599-602.

106. Schneider, R.; Zaitsev, A.G.; Fuchs, D.; v. Löhneysen, H. Superconductor-Insulator Quantum Phase Transition in Disordered FeSe Thin Films. Phys. Rev. Lett. 2012, 108, 257003. 
107. Speller, S.C.; Aksoy, C.; Saydam, M.; Taylor, H.; Burnell, G.; Boothroyd, A.T.; Grovenor, C.R.M. Analysis of $\mathrm{Fe}_{y} \mathrm{Se}_{1-x} \mathrm{Te}_{x}$ thin films grown by radio frequency sputtering. Supercond. Sci. Technol. 2011, 24, 075023.

108. Mousavi, T.; Grovenor, C.; Speller, S. Characterization of superconducting $\mathrm{Fe}_{y}\left(\mathrm{Se}_{1-x} \mathrm{Te}_{x}\right)$ thin films deposited on $\mathrm{MgO}$ substrates by sputtering. J. Mater. Sci. 2015, 50, 6970-6978.

109. Pawar, S.; Moholkar, A.; Suryavanshi, U.; Rajpure, K.; Bhosale, C. Electrosynthesis and characterization of iron selenide thin films. Sol. Energy Mater. Sol. Cells 2007, 91, 560-565.

110. Thanikaikarasan, S.; Mahalingam, T.; Sundaram, K.; Kathalingam, A.; Kim, Y.D.; Kim, T. Growth and characterization of electrosynthesized iron selenide thin films. Vacuum 2009, 83, 1066-1072.

111. Thanikaikarasan, S.; Mahalingam, T.; Raja, M.; Kim, T.; Kim, Y.D. Characterization of electroplated FeSe thin films. J. Mater. Sci. Mater. Electron. 2009, 20,727-734.

112. Chen, P.; Hu, S.; Liu, R.; Huang, C. Electrodeposition of nano-dimensioned FeSe. Thin Solid Films 2011, 519, 8397-8400.

113. Demura, S.; Ozaki, T.; Okazaki, H.; Mizuguchi, Y.; Kawasaki, Y.; Deguchi, K.; Watanabe, T.; Hara, H.; Yamaguchi, T.; Takeya, H.; et al. Electrochemical Synthesis of Iron-Based Superconductor FeSe Films. J. Phys. Soc. Jpn. 2012, 81, 043702.

114. Demura, S.; Okazaki, H.; Ozaki, T.; Hara, H.; Kawasaki, Y.; Deguchi, K.; Watanabe, T.; Denholme, S.J.; Mizuguchi, Y.; Yamaguchi, T.; et al. Electrodeposition as a new route to synthesize superconducting FeSe. Solid State Commun. 2013, 154, 40-42.

115. Li, L.; Yang, Z.R.; Sun, Y.P.; Zhang, J.Y.; Shen, D.Z.; Zhang, Y.H. Superconductivity and magnetism in FeSe thin films grown by metal-organic chemical vapor deposition. Supercond. Sci. Technol. 2011, 24, 015010.

116. Hussain, R.A.; Badshah, A.; Younis, A.; Khan, M.D.; Akhtar, J. Iron selenide films by aerosol assisted chemical vapor deposition from single source organometallic precursor in the presence of surfactants. Thin Solid Films 2014, 567, 58-63.

117. Eisterer, M.; Raunicher, R.; Weber, H.W.; Bellingeri, E.; Cimberle, M.R.; Pallecchi, I.; Putti, M.; Ferdeghini, C. Anisotropic critical currents in $\mathrm{FeSe}_{0.5} \mathrm{Te}_{0.5}$ films and the influence of neutron irradiation. Supercond. Sci. Technol. 2011, 24, 065016.

118. Mele, P.; Matsumoto, K.; Haruyama, Y.; Mukaida, M.; Yoshida, Y.; Ichino, Y.; Kiss, T.; Ichinose, A. In-field characterization of $\mathrm{FeTe}_{0.8} \mathrm{~S}_{0.2}$ epitaxial thin films with enhanced superconducting properties. Supercond. Sci. Technol. 2010, 23, 052001.

119. Iida, K.; Hänisch, J.; Reich, E.; Kurth, F.; Hühne, R.; Schultz, L.; Holzapfel, B.; Ichinose, A.; Hanawa, M.; Tsukada, I.; et al. Intrinsic pinning and the critical current scaling of clean epitaxial $\mathrm{Fe}(\mathrm{Se}, \mathrm{Te})$ thin films. Phys. Rev. B 2013, 87, 104510.

120. Braccini, V.; Kawale, S.; Reich, E.; Bellingeri, E.; Pellegrino, L.; Sala, A.; Putti, M.; Higashikawa, K.; Kiss, T.; Holzapfel, B.; et al. Highly effective and isotropic pinning in epitaxial $\mathrm{Fe}(\mathrm{Se}, \mathrm{Te})$ thin films grown on $\mathrm{CaF}_{2}$ substrates. Appl. Phys. Lett. 2013, 103, 172601.

121. Ozaki, T.; Wu, L.; Zhang, C.; Jaroszynski, J.; Si, W.; Zhou, J.; Zhu, Y.; Li, Q. A route for a strong increase of critical current in nanostrained iron-based superconductors. Nat. Commun. 2016, 7, 13036.

122. Han, Y.; Li, W.Y.; Cao, L.X.; Wang, X.Y.; Xu, B.; Zhao, B.R.; Guo, Y.Q.; Yang, J.L. Superconductivity in Iron Telluride Thin Films under Tensile Stress. Phys. Rev. Lett. 2010, 104, 017003.

123. Si, W.; Jie, Q.; Wu, L.; Zhou, J.; Gu, G.; Johnson, P.D.; Li, Q. Superconductivity in epitaxial thin films of $\mathrm{Fe}_{1.08} \mathrm{Te}: \mathrm{O}_{x}$. Phys. Rev. B 2010, 81, 092506.

124. Nie, Y.F.; Telesca, D.; Budnick, J.I.; Sinkovic, B.; Wells, B.O. Superconductivity induced in iron telluride films by low-temperature oxygen incorporation. Phys. Rev. B 2010, 82, 020508.

125. Tsukada, I.; Hanawa, M.; Komiya, S.; Ichinose, A.; Akiike, T.; Imai, Y.; Maeda, A. Mobility Analysis of FeTe Thin Films. J. Phys. Soc. Jpn. 2011, 80, 023712.

126. Narangammana, L.K.; Liu, X.; Nie, Y.F.; Rueckert, F.J.; Budnick, J.I.; Hines, W.A.; Gu, G.; Wells, B.O. Low temperature crystal structure and large lattice discontinuity at $T_{\mathrm{C}}$ in superconducting $\mathrm{FeTeO}_{x}$ films. Appl. Phys. Lett. 2013, 103, 102604.

127. Tsukada, I.; Hanawa, M.; Komiya, S.; Akiike, T.; Tanaka, R.; Imai, Y.; Maeda, A. Hall effect in superconducting $\mathrm{Fe}\left(\mathrm{Se}_{0.5} \mathrm{Te}_{0.5}\right)$ thin films. Phys. Rev. B 2010, 81, 054515.

128. Tsukada, I.; Nabeshima, F.; Ichinose, A.; Komiya, S.; Hanawa, M.; Imai, Y.; Maeda, A. Crossover from holeto electron-dominant regions in iron-chalcogenide superconductors induced by Te/Se substitution. Jpn. J. Appl. Phys. 2015, 54, 043102. 
129. Dimitrov, I.K.; Si, W.D.; Ku, W.; Han, S.J.; Jaroszynski, J. Unusual persistence of superconductivity against high magnetic fields in the strongly-correlated iron-chalcogenide film FeTe: $\mathrm{O}_{x}$. Low Temp. Phys. 2013, 39, 680-684.

130. Zhuang, J.; Yeoh, W.K.; Cui, X.; Xu, X.; Du, Y.; Shi, Z.; Ringer, S.P.; Wang, X.; Dou, S.X. Unabridged phase diagram for single-phased $\mathrm{FeSe}_{x} \mathrm{Te}_{1-x}$ thin films. Sci. Rep. 2014, 4, 7273.

131. Imai, Y.; Sawada, Y.; Nabeshima, F.; Maeda, A. Suppression of phase separation and giant enhancement of superconducting transition temperature in $\mathrm{FeSe}_{1-x} \mathrm{Te}_{x}$ thin films. Proc. Natl. Acad. Sci. USA 2015, 112, 1937-1940.

132. Imai, Y.; Sawada, Y.; Asami, D.; Nabeshima, F.; Maeda, A. Superconducting properties of FeSe $\mathrm{F}_{1-x} \mathrm{Te}_{x}$ films with. Physica C 2016, 530, 24.

133. Imai, Y.; Sawada, Y.; Nabeshima, F.; Asami, D.; Kawai, M.; Maeda, A. Control of structural transition in $\mathrm{FeSe}_{1-x} \mathrm{Te}_{x}$ thin films by changing substrate materials. Sci. Rep. 2017, 7, 46653.

134. Noji, T.; Hatakeda, T.; Hosono, S.; Kawamata, T.; Kato, M.; Koike, Y. Synthesis and post-annealing effects of alkaline-metal-ethylenediamine-intercalated superconductors $A_{x}\left(\mathrm{C}_{2} \mathrm{H}_{8} \mathrm{~N}_{2}\right)_{y} \mathrm{Fe}_{2-z} \mathrm{Se}_{2}(A=\mathrm{Li}, \mathrm{Na})$ with $T_{\mathrm{C}}=45 \mathrm{~K}$. Physica C 2014, 504, 8-11.

135. Hosono, S.; Noji, T.; Hatakeda, T.; Kawamata, T.; Kato, M.; Koike, Y. New Superconducting Phase of $\mathrm{Li}_{x}\left(\mathrm{C}_{6} \mathrm{H}_{16} \mathrm{~N}_{2}\right)_{y} \mathrm{Fe}_{2-z} \mathrm{Se}_{2}$ with $T_{\mathrm{C}}=41 \mathrm{~K}$ Obtained through the Post-Annealing. J. Phys. Soc. Jpn. 2016, $85,013702$.

136. Nabeshima, F.; Imai, Y.; Ichinose, A.; Tsukada, I.; Maeda, A. Growth and transport properties of FeSe/FeTe superlattice thin films. Jpn. J. Appl. Phys. 2017, 56, 020308.

137. Lubashevsky, Y.; Lahoud, E.; Chashka, K.; Podolsky, D.; Kanigel, A. Shallow pockets and very strong coupling superconductivity in $\mathrm{FeSe}_{x} \mathrm{Te}_{1-x}$. Nat. Phys. 2012, 8, 309-312.

138. Okazaki, K.; Ito, Y.; Ota, Y.; Kotani, Y.; Shimojima, T.; Kiss, T.; Watanabe, S.; Chen, C.T.; Niitaka, S.; Hanaguri, T.; et al. Superconductivity in an electron band just above the Fermi level: possible route to BCS-BEC superconductivity. Sci. Rep. 2014, 4, 4109.

139. Kasahara, S.; Watashige, T.; Hanaguri, T.; Kohsaka, Y.; Yamashita, T.; Shimoyama, Y.; Mizukami, Y.; Endo, R.; Ikeda, H.; Aoyama, K.; et al. Field-induced superconducting phase of FeSe in the BCS-BEC cross-over. Proc. Natl. Acad. Sci. USA 2014, 111, 16309-16313.

140. Tan, S.; Zhang, Y.; Xia, M.; Ye, Z.; Chen, F.; Xie, X.; Peng, R.; Xu, D.; Fan, Q.; Xu, H.; et al. Interface-induced superconductivity and strain-dependent spin density waves in $\mathrm{FeSe}_{\mathrm{SrTiO}}$ thin films. Nat. Mater. 2013, 12, 634-640.

141. He, S.; He, J.; Zhang, W.; Zhao, L.; Liu, D.; Liu, X.; Mou, D.; Ou, Y.B.; Wang, Q.Y.; Li, Z.; et al. Phase diagram and electronic indication of high-temperature superconductivity at $65 \mathrm{~K}$ in single-layer FeSe films. Nat. Mater. 2013, 12, 605-610.

142. Wen, H.Z.; Yi, S.; Jin, S.Z.; Fang, S.L.; Ming, H.G.; Yan, F.Z.; Hui, M.Z.; Jun, P.P.; Ying, X.; Hui, C.W.; et al. Direct Observation of High-Temperature Superconductivity in One-Unit-Cell FeSe Films. Chin. Phys. Lett. 2014, 31, 017401.

143. Deng, L.Z.; Lv, B.; Wu, Z.; Xue, Y.Y.; Zhang, W.H.; Li, F.S.; Wang, L.L.; Ma, X.C.; Xue, Q.K.; Chu, C.W. Meissner and mesoscopic superconducting states in 1-4 unit-cell FeSe films. Phys. Rev. B 2014, 90, 214513.

144. Zhang, Z.; Wang, Y.H.; Song, Q.; Liu, C.; Peng, R.; Moler, K.; Feng, D.; Wang, Y. Onset of the Meissner effect at $65 \mathrm{~K}$ in FeSe thin film grown on $\mathrm{Nb}$-doped $\mathrm{SrTiO}_{3}$ substrate. Sci. Bull. 2015, 60, 1301-1304.

145. Ge, J.F.; Liu, Z.L.; Liu, C.; Gao, C.L.; Qian, D.; Xue, Q.K.; Liu, Y.; Jia, J.F. Superconductivity above 100 K in single-layer FeSe films on doped $\mathrm{SrTiO}_{3}$. Nat. Mater. 2015, 14, 285-289.

146. Peng, R.; Shen, X.P.; Xie, X.; Xu, H.C.; Tan, S.Y.; Xia, M.; Zhang, T.; Cao, H.Y.; Gong, X.G.; Hu, J.P.; et al. Measurement of an Enhanced Superconducting Phase and a Pronounced Anisotropy of the Energy Gap of a Strained FeSe Single Layer in FeSe $/ \mathrm{Nb}: \mathrm{SrTiO}_{3} / \mathrm{KTaO}_{3}$ Heterostructures Using Photoemission Spectroscopy. Phys. Rev. Lett. 2014, 112, 107001.

147. Miyata, Y.; Nakayama, K.; Sugawara, K.; Sato, T.; Takahashi, T. High-temperature superconductivity in potassium-coated multilayer FeSe thin films. Nat. Mater. 2015, 14, 775-779.

148. Tang, C.; Zhang, D.; Zang, Y.; Liu, C.; Zhou, G.; Li, Z.; Zheng, C.; Hu, X.; Song, C.; Ji, S.; et al. Superconductivity dichotomy in K-coated single and double unit cell FeSe films on $\mathrm{SrTiO}_{3}$. Phys. Rev. B 2015, 92, 180507. 
149. Wen, C.H.P.; Xu, H.C.; Chen, C.; Huang, Z.C.; Lou, X.; Pu, Y.J.; Song, Q.; Xie, B.P.; Abdel-Hafiez, M.; Chareev, D.A.; et al. Anomalous correlation effects and unique phase diagram of electron-doped FeSe revealed by photoemission spectroscopy. Nat. Commun. 2016, 7, 10840.

150. Song, C.L.; Zhang, H.M.; Zhong, Y.; Hu, X.P.; Ji, S.H.; Wang, L.; He, K.; Ma, X.C.; Xue, Q.K. Observation of Double-Dome Superconductivity in Potassium-Doped FeSe Thin Films. Phys. Rev. Lett. 2016, 116, 157001.

151. Tang, C.; Liu, C.; Zhou, G.; Li, F.; Ding, H.; Li, Z.; Zhang, D.; Li, Z.; Song, C.; Ji, S.; et al. Interface-enhanced electron-phonon coupling and high-temperature superconductivity in potassium-coated ultrathin FeSe films on $\mathrm{SrTiO}_{3}$. Phys. Rev. B 2016, 93, 020507.

152. Zhang, W.H.; Liu, X.; Wen, C.H.P.; Peng, R.; Tan, S.Y.; Xie, B.P.; Zhang, T.; Feng, D.L. Effects of Surface Electron Doping and Substrate on the Superconductivity of Epitaxial FeSe Films. Nano Lett. 2016, 16, 1969-1973.

153. Shiogai, J.; Ito, Y.; Mitsuhashi, T.; Nojima, T.; Tsukazaki, A. Electric-field-induced superconductivity in electrochemically etched ultrathin FeSe films on $\mathrm{SrTiO}_{3}$ and $\mathrm{MgO}$. Nat. Phys. 2016, 12, 42-46.

154. Lei, B.; Cui, J.H.; Xiang, Z.J.; Shang, C.; Wang, N.Z.; Ye, G.J.; Luo, X.G.; Wu, T.; Sun, Z.; Chen, X.H. Evolution of High-Temperature Superconductivity from a Low- $T_{c}$ Phase Tuned by Carrier Concentration in FeSe Thin Flakes. Phys. Rev. Lett. 2016, 116, 077002.

155. Hanzawa, K.; Sato, H.; Hiramatsu, H.; Kamiya, T.; Hosono, H. Electric field-induced superconducting transition of insulating FeSe thin film at 35 K. Proc. Natl. Acad. Sci. USA 2016, 113, 3986-3990.

156. Saito, Y.; Nojima, T.; Iwasa, Y. Highly crystalline 2D superconductors. Nat. Rev. Mats. 2016, 2, 16094.

157. Lee, D.H. What makes the $T_{\mathrm{c}}$ of $\mathrm{FeSe} / \mathrm{SrTiO}_{3}$ so high? Chin. Phys. B 2015, 24, 117405.

158. Lee, J.J.; Schmitt, F.T.; Moore, R.G.; Johnston, S.; Cui, Y.T.; Li, W.; Yi, M.; Liu, Z.K.; Hashimoto, M.; Zhang, Y.; et al. Interfacial mode coupling as the origin of the enhancement of $T_{\mathrm{C}}$ in FeSe films on $\mathrm{SrTiO}_{3}$. Nature 2014, 515, 245-248.

159. Tian, Y.C.; Zhang, W.H.; Li, F.S.; Wu, Y.L.; Wu, Q.; Sun, F.; Zhou, G.Y.; Wang, L.; Ma, X.; Xue, Q.K.; et al. Ultrafast Dynamics Evidence of High Temperature Superconductivity in Single Unit Cell FeSe on $\mathrm{SrTiO}_{3}$. Phys. Rev. Lett. 2016, 116, 107001.

160. Zhang, S.; Guan, J.; Jia, X.; Liu, B.; Wang, W.; Li, F.; Wang, L.; Ma, X.; Xue, Q.; Zhang, J.; et al. Role of $\mathrm{SrTiO}_{3}$ phonon penetrating into thin FeSe films in the enhancement of superconductivity. Phys. Rev. B 2016, 94, 081116.

161. Peng, R.; Xu, H.C.; Tan, S.Y.; Cao, H.Y.; Xia, M.; Shen, X.P.; Huang, Z.C.; Wen, C.; Song, Q.; Zhang, T.; et al. Tuning the band structure and superconductivity in single-layer FeSe by interface engineering. Nat. Commun. 2014, 5, 5044.

162. Li, F.; Ding, H.; Tang, C.; Peng, J.; Zhang, Q.; Zhang, W.; Zhou, G.; Zhang, D.; Song, C.L.; He, K.; et al. Interface-enhanced high-temperature superconductivity in single-unit-cell $\mathrm{FeTe}_{1-x} \mathrm{Se}_{x}$ films on $\mathrm{SrTiO}_{3}$. Phys. Rev. B 2015, 91, 220503.

163. Zhou, G.; Zhang, D.; Liu, C.; Tang, C.; Wang, X.; Li, Z.; Song, C.; Ji, S.; He, K.; Wang, L.; Ma, X.; Xue, Q.K. Interface induced high temperature superconductivity in single unit-cell FeSe on $\mathrm{SrTiO}_{3}(110)$. Appl. Phys. Lett. 2016, 108, 202603.

164. Zhang, P.; Peng, X.L.; Qian, T.; Richard, P.; Shi, X.; Ma, J.Z.; Fu, B.B.; Guo, Y.L.; Han, Z.Q.; Wang, S.C.; et al. Observation of high- $T_{c}$ superconductivity in rectangular FeSe/SrTiO $3(110)$ monolayers. Phys. Rev. B 2016, 94, 104510.

165. Ding, H.; Lv, Y.F.; Zhao, K.; Wang, W.L.; Wang, L.; Song, C.L.; Chen, X.; Ma, X.C.; Xue, Q.K. High-Temperature Superconductivity in Single-Unit-Cell FeSe Films on Anatase $\mathrm{TiO}_{2}(001)$. Phys. Rev. Lett. 2016, 117, 067001.

166. Rebec, S.N.; Jia, T.; Zhang, C.; Hashimoto, M.; Lu, D.H.; Moore, R.G.; Shen, Z.X. Coexistence of Replica Bands and Superconductivity in FeSe Monolayer Films. Phys. Rev. Lett. 2017, 118, 067002.

167. Ginzburg, V. On surface superconductivity. Phys. Lett. 1964, 13, 101-102.

168. Fang, L.; Cheng, P.; Jia, Y.; Zhu, X.; Luo, H.; Mu, G.; Gu, C.; Wen, H.H. Growth of single crystals at ambient pressure and their transport properties. J. Cryst. Growth 2009, 311, 358-361.

169. Karpinski, J.; Zhigadlo, N.; Katrych, S.; Bukowski, Z.; Moll, P.; Weyeneth, S.; Keller, H.; Puzniak, R.; Tortello, M.; Daghero, D.; et al. Single crystals of $L n \mathrm{FeAsO}_{1-x} \mathrm{~F}_{x}(\mathrm{Ln}=\mathrm{La}, \mathrm{Pr}, \mathrm{Nd}, \mathrm{Sm}, \mathrm{Gd})$ and $\mathrm{Ba}_{1-x} \mathrm{Rb}_{x} \mathrm{Fe}_{2} \mathrm{As}_{2}$ : Growth, structure and superconducting properties. Physica C 2009, 469, 370-380.

170. Itoh, T.; Mori, M.; Inukai, M.; Nitani, H.; Yamamoto, T.; Miyanaga, T.; Igawa, N.; Kitamura, N.; Ishida, N.; Idemoto, Y. Effect of Annealing on Crystal and Local Structures of Doped Zirconia Using Experimental and Computational Methods. J. Phys. Chem. C 2015, 119, 8447-8458. 
171. Cao, X.Q.; Vassen, R.; Jungen, W.; Schwartz, S.; Tietz, F.; Stöver, D. Thermal Stability of Lanthanum Zirconate Plasma-Sprayed Coating. J. Am. Ceram. Soc. 2001, 84, 2086.

172. Diehl, R.; Brandt, G. Crystal structure refinement of $\mathrm{YAlO}_{3}$, a promising laser material. Mater. Res. Bull. 1975, 10, 85-90.

173. Chaix-Pluchery, O.; Chenevier, B.; Robles, J.J. Anisotropy of thermal expansion in $\mathrm{YAlO}_{3}$ and $\mathrm{NdGaO}_{3}$. Appl. Phys. Lett. 2005, 86, 251911.

174. Magrez, A.; Morniroli, J.; Caldes, M.; Marie, A.; Joubert, O.; Brohan, L. Using CBED and crystallographic image processing to evidence a structural distortion in a new family of ionic conductor $\mathrm{Sr}_{1-x} \mathrm{La}_{1+x} \mathrm{Al}_{1-x} \mathrm{Mg}_{x} \mathrm{O}_{4}(0 \leq x \leq 0.7)$. J. Solid State Chem. 2003, 172, 243-251.

175. Byszewski, P.; Domagała, J.; Fink-Finowicki, J.; Pajaczkowska, A. Thermal properties of CaNdAlO $\mathrm{Al}_{4}$ $\mathrm{SrLaAlO}_{4}$ single crystals. Mater. Res. Bull. 1992, 27, 483-490.

176. Lehnert, H.; Boysen, H.; Schneider, J.; Frey, F.; Hohlwein, D.; Radaelli, P.; Ehrenberg, H. A powder diffraction study of the phase transition in $\mathrm{LaAlO}_{3}$. Z. Kristallogr. 2000, 215, 536-541.

177. Chakoumakos, B.C.; Schlom, D.G.; Urbanik, M.; Luine, J. Thermal expansion of $\mathrm{LaAlO}_{3}$ and (La,Sr) $(\mathrm{Al}, \mathrm{Ta}) \mathrm{O}_{3}$ substrate materials for superconducting thin-film device applications. J. Appl. Phys. 1998, 83, 1979-1982.

178. Britten, J.F.; Dabkowska, H.A.; Dabkowski, A.B.; Greedan, J.E.; Campbell, J.L.; Teesdale, W.J. Czochralski-Grown SrLaGaO 4 . Acta Crystallogr. C 1995, 51, 1975-1977.

179. Hontsu, S.; Ishii, J.; Kawai, T.; Kawai, S. $\mathrm{LaSrGaO}_{4}$ substrate gives oriented crystalline $\mathrm{YBa}_{2} \mathrm{Cu}_{3} \mathrm{O}_{7-y}$ films. Appl. Phys. Lett. 1991, 59, 2886-2888.

180. Mateika, D.; Kohler, H.; Laudan, H.; Völkel, E. Mixed-perovskite substrates for high- $T_{\mathrm{C}}$ superconductors. J. Cryst. Growth 1991, 109, 441-446.

181. Nelmes, R.J.; Meyer, G.M.; Hutton, J. Thermal motion in $\mathrm{SrTiO}_{3}$ at room temperature: Anharmonic or disordered? Ferroelectrics 1978, 21, 461-462.

182. Touloukian, Y.; Kirby, R.; Taylor, E.; Lee, T. Thermophysical Properties of Matter-The TPRC Data Series. Volume 13. Thermal Expansion-Nonmetallic Solids; Defense Technical Information Center: Fort Belvoir, VA, USA, 1977.

183. Hazen, R.M. Effects of temperature and pressure on the cell dimension and X-ray temperature factors of periclas. Am. Mineral. 1976, 61, 266-271.

184. Cheetham, A.K.; Fender, B.E.F.; Cooper, M.J. Defect structure of calcium fluoride containing excess anions I. Bragg scattering. J. Phys. C Solid State Phys. 1971, 4, 3107.

185. Roberts, R.B.; White, G.K. Thermal expansion of fluorites at high temperatures. J. Phys. C Solid State Phys. 1986, 19, 7167 .

186. Forsyth, J.B.; Wilson, C.C.; Sabine, T.M. A time-of-flight neutron diffraction study of anharmonic thermal vibrations in $\mathrm{SrF}_{2}$, at the spallation neutron source ISIS. Acta Crystallogr. A 1989, 45, 244-247.

187. Radtke, S.; Brown, G.E. Frankdicksonite, $\mathrm{BaF}_{2}$, a new mineral from Nevada. Am. Mineral. 1974, 59, 885-888.

188. Stevenson, A.W. Thermal vibrations and bonding in GaAs: An extended-face crystal study. Acta Crystallogr. A 1994, 50, 621-632.

189. Uemura, H.; Kawaguchi, T.; Ohno, T.; Tabuchi, M.; Ujihara, T.; Takeda, Y.; Ikuta, H. Substrate dependence of the superconducting properties of $\operatorname{NdFeAs}(\mathrm{O}, \mathrm{F})$ thin films. Solid State Commun. 2012, 152, 735-739.

190. Takano, S.; Ueda, S.; Takeda, S.; Sugawara, H.; Naito, M. Substrate effect on structure and superconductivity in $\operatorname{SmFeAs}(\mathrm{O}, \mathrm{F})$ epitaxial films. Physica C 2012, 475, 10-13.

191. Hanawa, M.; Ichinose, A.; Komiya, S.; Tsukada, I.; Akiike, T.; Imai, Y.; Hikage, T.; Kawaguchi, T.; Ikuta, H.; Maeda, A. Substrate Dependence of Structural and Transport Properties in $\mathrm{FeSe}_{0.5} \mathrm{Te}_{0.5}$ Thin Films. Jpn. J. Appl. Phys. 2011, 50, 053101.

192. Iida, K.; Hänisch, J.; Tarantini, C.; Kurth, F.; Jaroszynski, J.; Ueda, S.; Naito, M.; Ichinose, A.; Tsukada, I.; Reich, E.; et al. Oxypnictide $\operatorname{SmFeAs}(\mathrm{O}, \mathrm{F})$ superconductor: A candidate for high-field magnet applications. Sci. Rep. 2013, 3, 2139.

193. Iida, K.; Grinenko, V.; Kurth, F.; Ichinose, A.; Tsukada, I.; Ahrens, E.; Pukenas, A.; Chekhonin, P.; Skrotzki, W.; Teresiak, A.; et al. Hall-plot of the phase diagram for $\mathrm{Ba}\left(\mathrm{Fe}_{1-x} \mathrm{Co}_{x}\right)_{2} \mathrm{As}_{2}$. Sci. Rep. 2016, 6, 28390.

194. Mizuguchi, Y.; Tomioka, F.; Tsuda, S.; Yamaguchi, T.; Takano, Y. Superconductivity at $27 \mathrm{~K}$ in tetragonal FeSe under high pressure. Appl. Phys. Lett. 2008, 93, 152505.

195. Masaki, S.; Kotegawa, H.; Hara, Y.; Tou, H.; Murata, K.; Mizuguchi, Y.; Takano, Y. Precise Pressure Dependence of the Superconducting Transition Temperature of FeSe: Resistivity and 77Se-NMR Study. J. Phys. Soc. Jpn. 2009, 78, 063704. 
196. Medvedev, S.; McQueen, T.M.; Troyan, I.A.; Palasyuk, T.; Eremets, M.I.; Cava, R.J.; Naghavi, S.; Casper, F.; Ksenofontov, V.; Wortmann, G.; et al. Electronic and magnetic phase diagram of $\beta$ - $\mathrm{Fe}_{1.01} \mathrm{Se}$ with superconductivity at $36.7 \mathrm{~K}$ under pressure. Nat. Mater. 2009, 8, 630.

197. Böhmer, A.E.; Hardy, F.; Eilers, F.; Ernst, D.; Adelmann, P.; Schweiss, P.; Wolf, T.; Meingast, C. Lack of coupling between superconductivity and orthorhombic distortion in stoichiometric single-crystalline FeSe. Phys. Rev. B 2013, 87, 180505.

198. Noji, T.; Suzuki, T.; Abe, H.; Adachi, T.; Kato, M.; Koike, Y. Growth, Annealing Effects on Superconducting and Magnetic Properties, and Anisotropy of $\mathrm{FeSe}_{1-x} \mathrm{Te}_{x}(0.5 \leq x \leq 1)$ Single Crystals. J. Phys. Soc. Jpn. 2010, $79,084711$.

199. McQueen, T.M.; Williams, A.J.; Stephens, P.W.; Tao, J.; Zhu, Y.; Ksenofontov, V.; Casper, F.; Felser, C.; Cava, R.J. Tetragonal-to-Orthorhombic Structural Phase Transition at $90 \mathrm{~K}$ in the Superconductor $\mathrm{Fe}_{1.01} \mathrm{Se}$. Phys. Rev. Lett. 2009, 103, 057002.

200. Li, S.; de la Cruz, C.; Huang, Q.; Chen, Y.; Lynn, J.; Hu, J.; Huang, Y.L.; Hsu, F.C.; Yeh, K.W.; Wu, M.K.; et al. First-order magnetic and structural phase transitions in $\mathrm{Fe}_{1+y} \mathrm{Se}_{x} \mathrm{Te}_{1-x}$. Phys. Rev. B 2009, 79, 054503.

201. Martinelli, A.; Palenzona, A.; Tropeano, M.; Ferdeghini, C.; Putti, M.; Cimberle, M.R.; Nguyen, T.D.; Affronte, M.; Ritter, C. From antiferromagnetism to superconductivity in $\mathrm{Fe}_{1+y} \mathrm{Te}_{1-x} \mathrm{Se}_{x}(0 \leq x \leq 0.20)$ : Neutron powder diffraction analysis. Phys. Rev. B 2010, 81, 094115.

202. Gresty, N.C.; Takabayashi, Y.; Ganin, A.Y.; McDonald, M.T.; Claridge, J.B.; Giap, D.; Mizuguchi, Y.; Takano, Y.; Kagayama, T.; Ohishi, Y.; et al. Structural Phase Transitions and Superconductivity in $\mathrm{Fe}_{1+\delta} \mathrm{Se}_{0.57} \mathrm{Te}_{0.43}$ at Ambient and Elevated Pressures. J. Am. Chem. Soc. 2009, 131, 16944.

203. Bao, W.; Qiu, Y.; Huang, Q.; Green, M.A.; Zajdel, P.; Fitzsimmons, M.R.; Zhernenkov, M.; Chang, S.; Fang, M.; Qian, B.; et al. Tunable $(\delta \pi, \delta \pi)$-Type Antiferromagnetic Order in $\alpha$-Fe(Te,Se) Superconductors. Phys. Rev. Lett. 2009, 102, 247001.

204. Wilson, S.D.; Yamani, Z.; Rotundu, C.R.; Freelon, B.; Bourret-Courchesne, E.; Birgeneau, R.J. Neutron diffraction study of the magnetic and structural phase transitions in $\mathrm{BaFe}_{2} \mathrm{As}_{2}$. Phys. Rev. B 2009, 79, 184519.

205. Misawa, T.; Imada, M. Superconductivity and its mechanism in an ab initio model for electron-doped LaFeAsO. Nat. Commun. 2014, 5, 6738.

206. Mendoza, D.; Benítez, J.; Morales, F.; Escudero, R. Magnetic anomaly in superconducting FeSe. Solid State Commun. 2010, 150, 1124.

207. Song, Y.J.; Hong, J.B.; Min, B.H.; Kwon, Y.S.; Lee, K.J.; Jung, M.H.; Rhyee, J.S. Superconducting Properties of a Stoichiometric FeSe Compound and Two Anomalous Features in the Normal State. J. Korean Phys. Soc. 2011, 59, 312, doi:10.3938/jkps.59.312.

208. Phan, G.N.; Nakayama, K.; Sugawara, K.; Sato, T.; Urata, T.; Tanabe, Y.; Tanigaki, K.; Nabeshima, F.; Imai, Y.; Maeda, A.; et al. Effects of strain on the electronic structure, superconductivity, and nematicity in FeSe studied by angle-resolved photoemission spectroscopy. Phys. Rev. B 2017, 95, 224507.

209. Sawada, Y.; Nabeshima, F.; Imai, Y.; Maeda, A. Investigation of Transport Properties for FeSe $e_{1-x} \mathrm{Te}_{x} \mathrm{Thin}$ Films under Magnetic Fields. J. Phys. Soc. Jpn. 2016, 85, 073703.

210. Horigane, K.; Hiraka, H.; Ohoyama, K. Relationship between Structure and Superconductivity in $\mathrm{FeSe}_{1-x} \mathrm{Te}_{x}$. J. Phys. Soc. Jpn. 2009, 78, 074718.

211. Izumi, F.; Momma, K. Three-Dimensional Visualization in Powder Diffraction. Solid State Phenom. 2007, $130,15-20$.

212. Mizuguchi, Y.; Hara, Y.; Deguchi, K.; Tsuda, S.; Yamaguchi, T.; Takeda, K.; Kotegawa, H.; Tou, H.; Takano, Y. Anion height dependence of $T_{\mathrm{C}}$ for the Fe-based superconductor. Supercond. Sci. Technol. 2010, 23, 054013.

213. Sawada, Y.; Nabeshima, F.; Asami, D.; Ogawa, R.; Imai, Y.; Maeda, A. Transport properties of FeSe $\mathrm{Fe}_{1-x} \mathrm{Te}_{x}$ thin films under magnetic fields up to 8 T. Physica C 2016, 530, 27-30.

214. Nakajima, M.; Yanase, K.; Nabeshima, F.; Imai, Y.; Maeda, A.; Tajima, S. Gradual Fermi-surface modification in orbitally ordered state of FeSe revealed by optical spectroscopy. Phys. Rev. B 2017, 95, 184502.

215. Sun, Y.; Pyon, S.; Tamegai, T. Electron carriers with possible Dirac-cone-like dispersion in FeSe ${ }_{1-x} \mathrm{~S}_{x}(x=0$ and 0.14) single crystals triggered by structural transition. Phys. Rev. B 2016, 93, 104502.

216. Miyoshi, K.; Morishita, K.; Mutou, E.; Kondo, M.; Seida, O.; Fujiwara, K.; Takeuchi, J.; Nishigori, S. Enhanced Superconductivity on the Tetragonal Lattice in FeSe under Hydrostatic Pressure. J. Phys. Soc. Jpn. 2014, $83,013702$. 
217. Sun, J.P.; Matsuura, K.; Ye, G.Z.; Mizukami, Y.; Shimozawa, M.; Matsubayashi, K.; Yamashita, M.; Watashige, T.; Kasahara, S.; Matsuda, Y.; et al. Dome-shaped magnetic order competing with high-temperature superconductivity at high pressures in FeSe. Nat. Commun. 2016, 7, 12146.

218. Kothapalli, K.; Böhmer, A.E.; Jayasekara, W.T.; Ueland, B.G.; Das, P.; Sapkota, A.; Taufour, V.; Xiao, Y.; Alp, E.; Bud'ko, S.L.; et al. Strong cooperative coupling of pressure-induced magnetic order and nematicity in FeSe. Nat. Commun. 2016, 7, 12728.

219. Hosoi, S.; Matsuura, K.; Ishida, K.; Wang, H.; Mizukami, Y.; Watashige, T.; Kasahara, S.; Matsuda, Y.; Shibauchi, T. Nematic quantum critical point without magnetism in $\mathrm{FeSe}_{1-x} \mathrm{~S}_{x}$ superconductors. Proc. Natl. Acad. Sci. USA 2016, 113, 8139-8143.

220. Watson, M.D.; Kim, T.K.; Haghighirad, A.A.; Blake, S.F.; Davies, N.R.; Hoesch, M.; Wolf, T.; Coldea, A.I. Suppression of orbital ordering by chemical pressure in $\mathrm{FeSe}_{1-x} \mathrm{~S}_{x}$. Phys. Rev. B 2015, 92, 121108.

221. Yoshizawa, M.; Kimura, D.; Chiba, T.; Simayi, S.; Nakanishi, Y.; Kihou, K.; Lee, C.H.; Iyo, A.; Eisaki, H.; Nakajima, M.; et al. Structural Quantum Criticality and Superconductivity in Iron-Based Superconductor $\mathrm{Ba}\left(\mathrm{Fe}_{1-x} \mathrm{Co}_{x}\right)_{2} \mathrm{As}_{2}$. J. Phys. Soc. Jpn. 2012, 81, 024604.

222. Kuo, H.H.; Chu, J.H.; Palmstrom, J.C.; Kivelson, S.A.; Fisher, I.R. Ubiquitous signatures of nematic quantum criticality in optimally doped Fe-based superconductors. Science 2016, 352, 958-962.

223. Lederer, S.; Schattner, Y.; Berg, E.; Kivelson, S.A. Enhancement of Superconductivity near a Nematic Quantum Critical Point. Phys. Rev. Lett. 2015, 114, 097001.

224. Bendele, M.; Ichsanow, A.; Pashkevich, Y.; Keller, L.; Strässle, T.; Gusev, A.; Pomjakushina, E.; Conder, K.; Khasanov, R.; Keller, H. Coexistence of superconductivity and magnetism in $\mathrm{FeSe}_{1-x}$ under pressure. Phys. Rev. B 2012, 85, 064517.

225. Terashima, T.; Kikugawa, N.; Kasahara, S.; Watashige, T.; Shibauchi, T.; Matsuda, Y.; Wolf, T.; Böhmer, A.E.; Hardy, F.; Meingast, C.; et al. Pressure-Induced Antiferromagnetic Transition and Phase Diagram in FeSe. J. Phys. Soc. Jpn. 2015, 84, 063701.

226. Nabeshima, F.; Nagasawa, K.; Imai, Y.; Maeda, A. Superconducting fluctuations in $\mathrm{FeSe}_{0.5} \mathrm{Te}_{0.5}$ thin films probed via microwave spectroscopy. arXiv 2017, arXiv:1706.06727.

(C) 2017 by the authors. Licensee MDPI, Basel, Switzerland. This article is an open access article distributed under the terms and conditions of the Creative Commons Attribution (CC BY) license (http:/ / creativecommons.org/licenses/by/4.0/). 